\title{
Differential Entrainment and Learning-Related Dynamics of Spike and Local Field Potential Activity in the Sensorimotor and Associative Striatum
}

\author{
Catherine A. Thorn ${ }^{1,2}$ and Ann M. Graybiel ${ }^{2,3}$ \\ ${ }^{1}$ Department of Electrical Engineering and Computer Science, ${ }^{2} \mathrm{McGovern}$ Institute for Brain Research, and ${ }^{3}$ Department of Brain and Cognitive Sciences, \\ Massachusetts Institute of Technology, Cambridge, Massachusetts 02139
}

Parallel cortico-basal ganglia loops are thought to have distinct but interacting functions in motor learning and habit formation. In rats, the striatal projection neuron populations (MSNs) in the dorsolateral and dorsomedial striatum, respectively corresponding to sensorimotor and associative regions of the striatum, exhibit contrasting dynamics as rats acquire T-maze tasks (Thorn et al., 2010). Here, we asked whether these patterns could be related to the activity of local interneuron populations in the striatum and to the local field potential activity recorded simultaneously in the corresponding regions. We found that dorsolateral and dorsomedial striatal fastspiking interneurons exhibited task-specific and training-related dynamics consistent with those of corresponding MSN populations. Moreover, both MSNs and interneuron populations in both regions became entrained to theta-band (5-12 Hz) frequencies during task acquisition. However, the predominant entrainment frequencies were different for the sensorimotor and associative zones. Dorsolateral striatal neurons became entrained mid-task to oscillations centered $\sim 5 \mathrm{~Hz}$, whereas simultaneously recorded neurons in the dorsomedial region became entrained to higher frequency $(\sim 10 \mathrm{~Hz})$ rhythms. These region-specific patterns of entrainment evolved dynamically with the development of region-specific patterns of interneuron and MSN activity, indicating that, with learning, these two striatal regions can develop different frequency-modulated circuit activities in parallel. We suggest that such differential entrainment of sensorimotor and associative neuronal populations, acquired through learning, could be critical for coordinating information flow throughout each trans-striatal network while simultaneously enabling nearby components of the separate networks to operate independently.

Key words: dorsolateral; dorsomedial; electrophysiology; habit learning; oscillation; striatum

\section{Introduction}

The basal ganglia are part of forebrain systems that control the expression of motor and cognitive behavioral sequences and their acquisition through reinforcement-based learning. Different cortico-basal ganglia circuits have been proposed to underpin the motor, associative and limbic functions of the basal ganglia, and yet the operation of these loops is still incompletely understood. In work in rodent species, a distinction is made between the dorsolateral and dorsomedial parts of the striatum, which correspond, respectively, to sensorimo-

Received April 25, 2013; revised Jan. 6, 2014; accepted Jan. 11, 2014.

Author contributions: C.A.T. and A.M.G. designed research; C.A.T. performed research; C.A.T. and A.M.G. analyzed data; C.A.T. and A.M.G. wrote the paper.

This work was supported by the National Institutes of Health Grant MH060379; Office of Naval Research Grant N00014-04-1-0208; National Science Foundation Grant NSF-DMS-1042134; the Stanley H. and Sheila G. Sydney Fund; European Union Grant 201716; and the McGovern Institute for Brain Research fellowship to C.A.T. We thank Ira Jaffe, R. Rae Pourian, Julia Madadi, and Bennett Zarren for their generous support; and Patricia Harlan, Christine Keller-McGandy, Henry Hall, and Alexander McWhinnie for their help.

The authors declare no competing financial interests.

Correspondence should be addressed to Dr. Ann M. Graybiel, Massachusetts Institute of Technology, 46-6133, 43 Vassar Street, Cambridge, MA 02139. E-mail: graybiel@mit.edu.

C.A. Thorn's present address: Department of Neuroscience, Division of Biology and Medicine, Brown University, Providence, Rhode Island.

DOI:10.1523/JNEUROSCI.1782-13.2014

Copyright $\odot 2014$ the authors $\quad 0270-6474 / 14 / 342845-15 \$ 15.00 / 0$ tor and associative zones. These subregions have been shown to be engaged differentially during habitual and flexible behaviors: the sensorimotor zone must be intact for habitual performance, and the associative zone for flexibility (Yin et al., 2006; Packard, 2009; Balleine and O'Doherty, 2010). Neurons in these two regions exhibit distinct activities during the learning and performance of sensorimotor tasks (Berke et al., 2004; Kimchi et al., 2009; Yin et al., 2009). Moreover, the projection neuron populations (MSNs) in these dorsolateral and dorsomedial striatal regions exhibit distinct dynamics in their activities both across the execution of the behavioral sequence and during the course of learning (Thorn et al., 2010).

The origin of these region-specific differences in the activity remains unclear. The sensorimotor and associative striatal regions receive distinct cortical, thalamic, and neuromodulatory inputs (Alexander et al., 1986; McGeorge and Faull, 1989; Haber, 2003; Voorn et al., 2004). Additional variation, however, could arise from differences in the local processing of input-output mappings within each region. Here we asked, first, whether interneurons in these two striatal regions exhibit population dynamics that could shape the activity of simultaneously recorded MSNs by examining the activity of key striatal interneuron subtypes: the fast-spiking interneurons (FSIs, putative parvalbumincontaining interneurons) and the tonically active interneurons 
(TANs, putative cholinergic interneurons). We tracked their activities by recording with chronically implanted electrodes as rats acquired and were overtrained on a T-maze task.

Second, given that local field potentials (LFPs) can reflect synaptic activity and input structure within a region (Logothetis, 2002; Buzsáki et al., 2012) and thus could offer a window into the influence of network inputs on striatal processing, we asked whether sensorimotor and associative striatal circuits could be distinguished according to differential entrainment of local single neurons to ongoing oscillations. Such rhythmic activities have been hypothesized to play a role in synchronizing information transfer through cortico-basal ganglia loops (Gatev et al., 2006; Kojima et al., 2013), and entrainment of single-unit firing to the LFPs suggests that ongoing oscillations are indeed relevant to striatal processing (DeCoteau et al., 2007a; Tort et al., 2008; Berke, 2009; Burkhardt et al., 2009; Kimchi et al., 2009; Sharott et al., 2009; van der Meer and Redish, 2009; Kalenscher et al., 2010; Howe et al., 2011; Malhotra et al., 2012). Here, we demonstrate that not only do the spiking patterns of interneurons and projection neurons differ between these two key striatal regions, but also the predominant frequency of entrainment of their spiking to theta-band oscillations are distinct and distinguish the concurrent activities of the sensorimotor and associative striatum as they are shaped during habit learning.

\section{Materials and Methods}

The dataset reported here was collected along with the data reported for MSN activity in Thorn et al. (2010). The MSN data were completely reanalyzed, and simultaneously recorded FSI, TAN, and LFP data were subjected to first analysis here together with the newly analyzed MSN activities. All experimental procedures were approved by the Committee on Animal Care at the Massachusetts Institute of Technology.

\section{Experimental procedures}

The surgical, behavioral, and histological procedures are described in detail in our previous report. Briefly, nine adult male Long-Evans rats were implanted with headstages containing 12 tetrodes lowered to recording sites in dorsolateral $(\mathrm{AP}=0.5 \pm 0.5 \mathrm{~mm}, \mathrm{ML}=3.5 \pm 0.5 \mathrm{~mm}$, $\mathrm{DV}=3.5$ to $5.25 \mathrm{~mm})$ and dorsomedial $(\mathrm{AP}=1.7 \pm 0.5 \mathrm{~mm}, \mathrm{ML}=$ $1.8 \pm 0.5 \mathrm{~mm}, \mathrm{DV}=3.75$ to $5.5 \mathrm{~mm}$ ) regions of the caudoputamen (striatum) before behavioral training (see Fig. 1A). During training, rats concurrently acquired two versions of a T-maze task (see Fig. $1 B, C$ ). In the auditory version of the task, one of two tones $(1$ or $8 \mathrm{kHz})$ indicated the direction of the baited goal arm; in the tactile version, two tactile floor textures (rough or smooth runway insert) were used. The animals performed 80 trials per daily training session, in interleaved sets of 20 trials per modality. Within each set, stimuli instructing each turn direction were presented in pseudorandom order, and the starting modality was alternated daily. Training ended after 10 overtraining sessions were performed. Group 1 rats $(n=6)$ were trained on a difficult version of the tactile discrimination, which they failed to acquire, and overtraining ended for these rats after 10 consecutive sessions in which performance on the auditory version was significantly above chance $(>72.5 \%$ correct, $p<0.01, \chi^{2}$ test). Group 2 rats $(n=3)$ acquired an easier version of the tactile discrimination, and overtraining ended for these animals after 10 consecutive sessions in which performance on both versions was above chance. After the conclusion of training, animals were lightly anesthetized, and small lesions were made by passing current $(25 \mu \mathrm{A}, 10 \mathrm{~s})$ through each tetrode to mark the tip of the recording tracks. One to three days later, animals were anesthetized with a lethal dose of sodium pentobarbital and were perfused transcardially with $4 \%$ paraformaldehyde in $0.1 \mathrm{M} \mathrm{NaKPO}_{4}$ buffer. Transverse sections were cut, stained for Nissl substance, and examined microscopically to identify tetrode tracks and lesions. One of the animals in Group 1 (D11) was excluded from data analysis after histological examination showed tetrode placement to be $>0.5 \mathrm{~mm}$ anterior to the targeted striatal locations.

\section{Data acquisition and neuron classification}

Single-unit and LFP activity was recorded simultaneously throughout training using a Cheetah Data Acquisition System (Neuralynx). We relied on an overhead CCD camera to track the position of an LED affixed to the head of the rat, and we also registered photobeam breaks to detect maze behavioral events. Input signals from unit firing were processed (amplified at gains of 1000-10,000 and filtered at 600-6000 Hz), and these signals were then sampled at 30 or $32 \mathrm{kHz}$ for $\sim 1 \mathrm{~ms}$ ( 1.056 or 0.998 $\mathrm{ms}$ ) around the time of threshold crossing. We sorted recorded spikes manually into different clusters (units) with Offline Sorter (Plexon). For initial analysis of the spike data, we manually classified clusters and graded each for cluster quality. Because interneurons make up only a small percentage of striatal neurons, we established highly stringent criteria to minimize the inclusion of inadequately isolated or potentially misclassified units in the current report. We reexamined the spike firing of our entire database of 6750 single units recorded throughout training from both dorsolateral and dorsomedial regions of the striatum and used strict cluster quality and classification criteria based on published methods (Berke et al., 2004; Schmitzer-Torbert et al., 2005; Schmitzer-Torbert and Redish, 2008). Clusters were initially accepted for further analysis if they contained at least 150 spikes and were recorded during a session in which at least 35 trials were performed. Cluster quality was assessed using LRatio criteria as described by Schmitzer-Torbert et al. (2005). Putative FSIs and putative MSNs were further identified by applying a $k$-means clustering algorithm (MATLAB's kmeans command) to group units based on three parameters: peak width, valley width at half-minimum, and firing rate for each unit (see Fig. 1D). Thresholds were determined by the $k$-means algorithm, and any borderline cases were excluded. Units identified as FSIs were those with short spike durations (peak width $<$ $200 \mu \mathrm{s}$; valley width at half minimum $<326 \mu$ s) and mean firing rates $>6.95 \mathrm{~Hz}$ (population median firing rate $=17.9 \mathrm{~Hz}$ ), and they were accepted for further analysis if LRatio $<0.1$. Putative MSNs had longer spike durations (peak width $<200 \mu \mathrm{s} ; 320 \mu \mathrm{s}<$ valley width at halfminimum $<660 \mu \mathrm{s}$ ) and firing rates $<10 \mathrm{~Hz}$ (population median $=$ $1.19 \mathrm{~Hz}$ ), and were accepted for analyses if LRatio $<0.1$.

Clusters representing TANs were in general less well isolated and were therefore accepted for further analysis with LRatio $<0.3$. Further, TANs could not be distinguished completely from FSI and MSN subtypes on the basis of wave-shape criteria. This group of neurons was, instead, identified according to their firing properties (see Fig. $1 E$ ), including very low percentages of spikes occurring in bursts $(<2.5 \%)$, long postspike suppression $(>45 \mathrm{~ms})$, and relatively low overall firing rates, evidenced by a small percentage of interspike intervals (ISIs) of $<100 \mathrm{~ms}$ duration $(<7.5 \%)$. Thresholds were set by the experimenter, and clusters with parameters exceeding any threshold were excluded. Each cluster classified as a putative TAN was verified additionally by visual inspection post hoc. For the purposes of TAN classification, a "burst" was defined as a series of at least two spikes in which the ISIs were $<10 \mathrm{~ms}$. Postspike suppression is a pseudo-measure of refractory period previously developed by Schmitzer-Torbert and Redish (2008). The measure was computed by first constructing the $0-1 \mathrm{~s}$ autocorrelation histogram for each unit using $1 \mathrm{~ms}$ bins. The autocorrelogram was then smoothed by convolution with a 50-point Hamming window, and the postspike suppression was calculated as the time at which firing first exceeded the average firing rate over the $1 \mathrm{~s}$ window.

Clusters of spikes recorded in consecutive sessions were identified as putatively arising from the same neuron based on correlations between (1) the wave-shapes recorded on all four tetrode channels (Emondi et al., 2004), (2) the task-related pattern of activity constructed for the two clusters, and (3) the ISI and autocorrelation histograms (for FSI and MSN subtypes). Correlation coefficient thresholds were set separately for each unit subtype to minimize false repeats (Table 1), and all putative repeats were verified by visual inspection before acceptance.

LFPs were recorded simultaneously with the single-unit activity (gain: 1000, filtered: $1-475 \mathrm{~Hz}$, sampling rate: 1 or $1.89 \mathrm{kHz}$ ). LFPs from one Group 1 animal (D15) were contaminated by high levels of noise and were excluded from the dataset. 
Table 1. Correlation coefficients used to identify single units recorded across multiple sessions

\begin{tabular}{lllll}
\hline & $R_{\text {waveshape }}$ & $R_{\text {trialActivity }}$ & $R_{\text {ISI }}$ & $R_{\text {autocorr }}$ \\
\hline MSN & 0.98 & 0.9 & 0.98 & 0.7 \\
FSI & 0.98 & 0.75 & 0.98 & 0.7 \\
TAN & 0.98 & 0.75 & NA & NA \\
\hline
\end{tabular}

\section{Data analysis}

Normalization of single-unit and population activity. For each unit, perievent time histograms (PETHs) were created using $20 \mathrm{~ms}$ bins and $\pm 300 \mathrm{~ms}$ windows around each of 9 task events: baseline (BL), warning click (W), gate opening (Ga), locomotion onset (L), out-of-start (S), cue onset (C), turn start (TS), turn end (TE), and goal-reaching (Go). For these analyses, the baseline period was defined as the $600 \mathrm{~ms}$ window centered at $1.5 \mathrm{~s}$ before the presentation of the warning click denoting trial-start. Two methods were then used to normalize the firing rate of each unit. For direct comparison to our previous work, activity in each bin, $\mathrm{S}_{\text {bin }}$, was $z$-score normalized using the mean, $\mathrm{S}_{\text {mean }}$, and $\mathrm{SD}, \mathrm{S}_{\text {std }}$, computed over all 261 bins $(9$ events $\times 29$ bins/event $): Z_{\text {bin }}=\left(S_{\text {bin }}-\right.$ $\left.\mathrm{S}_{\text {mean }}\right) / \mathrm{S}_{\text {std }}$. The average $z$-score normalized population activity was then computed for each bin by taking the mean across all units included in a population and smoothing with a 3-point moving average filter. For the quantitative analyses in the current report, the concatenated PETHs representing the task-related activity of each unit were also 0-1 (minimummaximum) scaled such that normalized activity in each bin was nonnegative. The specific normalization method used was not found to impact significantly the results of the analyses.

Classification of task-responsive units. Units were determined to be task-responsive according to methods similar to those described previously (Barnes et al., 2005). Briefly, PETHs were constructed as described above ( $\pm 300 \mathrm{~ms}$ windows, $20 \mathrm{~ms}$ bins) and were concatenated to represent firing activity across task-time for each unit. If the firing rate in any of the perievent windows exceeded 2 SDs above or below the mean baseline firing rate for 4 consecutive $20 \mathrm{~ms}$ bins, the unit was classified as task-responsive.

To characterize the firing of TANs in greater detail, we performed additional analyses to identify phasic responses at goal-reaching, as well as the existence of burst, pause, and rebound phases of responding to the presentation of the warning click denoting trial-start and the onset of the conditional cue mid-maze. For each TAN, the spike train was first converted to a continuous waveform and sampled at the same rate as the simultaneously recorded LFP by placing a 1 at the sample closest to the time of the spike and 0 at all other samples. The continuous spike signal was then smoothed using an $\mathrm{N}$-point moving average filter, where $\mathrm{N}$ was equal to the sampling rate of the recorded LFP divided by 8 , such that the smoothing window width was $\sim 125 \mathrm{~ms}$. The mean firing rates were then calculated for $\pm 1 \mathrm{~s}$ perievent windows around warning click, cue onset, and goal-reaching events, and confidence limits were estimated for each time point as $2 \times$ SEM. For warning click and cue onset events, a "pause" was identified if the firing rate during a $1 \mathrm{~s}$ window after the event fell significantly below the pre-event firing rate. If the firing rate then rose to a level significantly above the pre-event rate within the $1 \mathrm{~s}$ window, a "rebound" response was identified. "Burst" responses were noted if the firing rate was elevated above the pre-event rate before the occurrence of a pause or, if no pause were found, within $600 \mathrm{~ms}$ of the event onset. Around goal-reaching, a response was determined to have occurred if the firing rate during the $1 \mathrm{~s}$ interval after the event significantly differed from the pre-event rate. For all tests, the low and high thresholds for significant responding were defined as the average lower and upper $95 \%$ confidence limits, respectively, over a $250 \mathrm{~ms}$ window before the event.

Modulation index (MI). For each unit, a MI was calculated to characterize the relationship between the unit's firing rate mid-maze and its pre- and post-trial firing rates. Firing across the entire task-time was represented by concatenating PETHs (20 ms bins) constructed for each of the 9 task events ( $\pm 300 \mathrm{~ms}$ perievent windows). The concatenated task activity was then normalized to a $0-1$ (minimum-maximum) scale. Intask firing rate, $F R_{I N}$, was calculated as the mean over the concatenated
PETH bins for the period beginning when the animals exited the start box (out-of-start event, S) through the end of the window centered on turn start (TS). Out-of-task firing rate, $F R_{\text {OUT }}$, was calculated as the mean over the concatenated PETH bins from the beginning of the baseline period (BL) to the onset of the warning click denoting trial start (W) in addition to the $300 \mathrm{~ms}$ period after goal-reaching. The final $\mathrm{MI}$ for each unit was then calculated as $M I=\left(F R_{I N}-F R_{O U T}\right) /\left(F R_{I N}+F R_{\text {OUT }}\right)$. A positive $\mathrm{MI}$ thus indicates a higher firing rate in-task compared with out-of-task, whereas a negative MI indicates the opposite.

Learning-related changes in population activity. To evaluate changes in population activity across training, PETHs were first constructed as described above ( $\pm 300 \mathrm{~ms}$ windows, $20 \mathrm{~ms}$ bins) around each of the 9 task events and were concatenated to represent activity across task-time for each unit. Task-related activity was then normalized to a $0-1$ (minimum-maximum) scale. For each training block, normalized activity for all units recorded during that block in each region was averaged across units to obtain the task-related response of the population. The uniformity of firing activity across task-time was then characterized for the population by measuring the coefficient of variation (CV) across all bins of the population-averaged normalized activity. The mean and $95 \%$ confidence limits of the CV measure were found by drawing 1000 bootstrap samples from the population of units recorded in each region during each training block. For Blocks B and C, the nonuniformity of firing across task-time was considered significantly greater than that in Block $\mathrm{A}$ if an estimated bootstrap $p$ value was found to be $<0.05: p=\sum_{i=1: 1000}$ $\left(C V_{A, i}>C V_{X, i}\right) / 1000$, where $C V_{X, i}$ denotes the coefficient of variation for $X=$ Blocks B and $\mathrm{C}$ and $i=$ bootstrap sample 1 to 1000 .

To compare the click-evoked response of the TAN populations early in training to that of TAN populations late in training, we constructed 1000 sample spike populations from the recorded data, for each region (dorsolateral, dorsomedial) and for each training condition (early, late). For each rat, "Early" training sessions included the first 10 sessions in which at least 35 trials were performed and performance on both tasks remained $<72.5 \%$ correct. For animals that acquired the auditory task version in $<10$ sessions, as many sessions as met these criteria were included. "Late" training sessions consisted of the last 10 sessions of training for Group 1 animals (Block B), and the last 20 sessions of training for Group 2 animals (sessions from Blocks B and C). To construct each sample spike population, a $\pm 300 \mathrm{~ms}$ window around warning click was considered, and 150 perievent spikes were randomly selected from each neuron in the population. A perievent histogram was then constructed for each of the 1000 population spike samples ( $40 \mathrm{~ms}$ bins) and normalized by the sum of spikes across all bins to create a spike probability distribution across the perievent window. A significant difference from uniform firing was considered to exist if the $95 \%$ confidence limits for any bin differed from the expected firing probability for a uniform distribution containing 15 bins (equal to $1 / 15$, or 0.0667 ). A bootstrap $p$ value, $p_{b i n}$, was estimated for each bin as the minimum of $\left[q_{b i n}, 1-q_{b i n}\right]$, where $q_{b i n}=\sum_{i=1: 1000}\left(H_{\text {norm } X, i}(\right.$ bin $\left.)>0.0667\right) / 1000$, and $p$ was taken to be the minimum $p_{b i n}$ across all bins.

Dorsolateral-dorsomedial striatal LFP coherence and spike-LFP coherence. The multitaper method was used to calculate LFP and spike-LFP coherence (Pesaran et al., 2002), according to procedures described previously (DeCoteau et al., 2007a). Coherograms in the current study were constructed by plotting coherence during a series of overlapping $1 \mathrm{~s}$ windows, using 2 tapers, using a smoothing width of 3 and padding each signal with zeros to three times its original length.

LFP-spike entrainment. For each unit, we tested whether a significant entrainment to the LFP existed for frequencies between 3 and $15 \mathrm{~Hz}$. For each rat, the two LFP channels in each region that contained the least noise and clipping artifacts across all sessions were used for this analysis. For each unit, the paired LFP was chosen as the best of these 4 as long as it was recorded in the same region but not on the same tetrode. The selected LFP was first bandpass filtered using a $5 \mathrm{~Hz}$ wide band, centered on frequencies from 3 to $15 \mathrm{~Hz}$, in $0.5 \mathrm{~Hz}$ increments. A Hilbert transform was then performed on the filtered LFP to obtain the continuous phase of the LFP throughout recording at the chosen frequency, and periods of clipping in the LFP data were removed. We quantified phase consistency using both the Rayleigh test of circular uniformity and the 

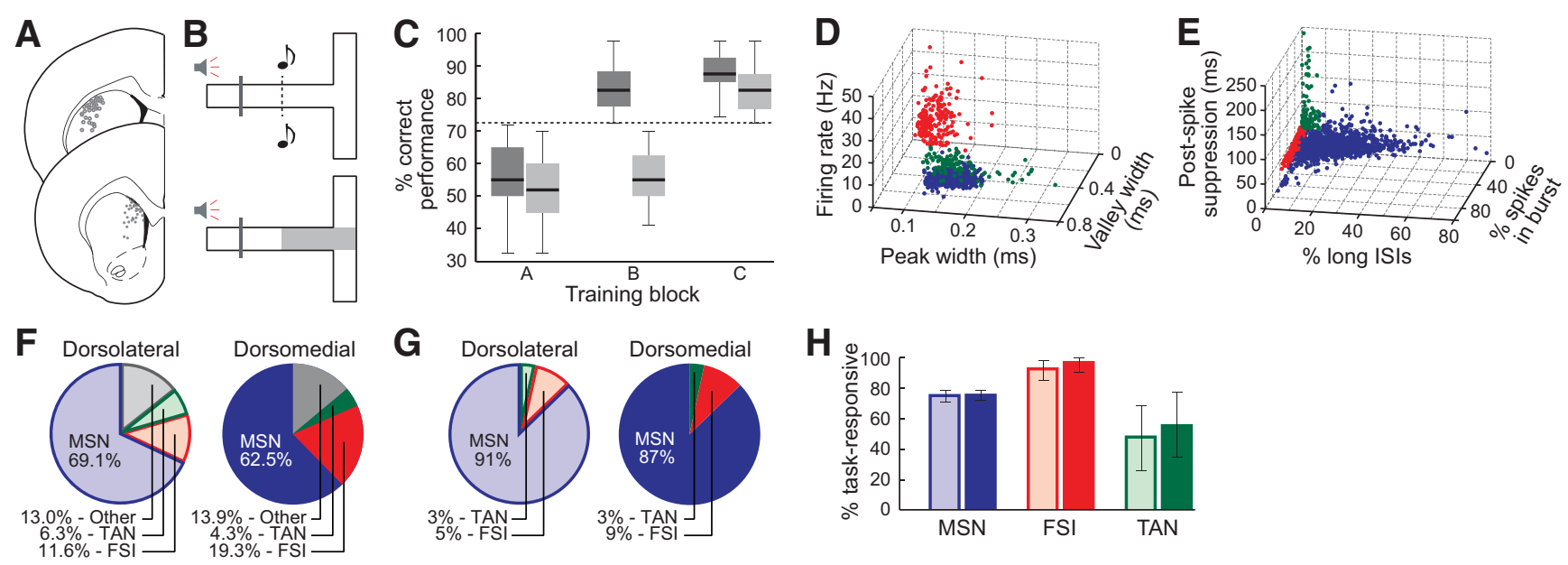

Figure 1. Behavioral task and single-unit recording. $\boldsymbol{A}$, Histologically identified locations of tetrode tips in dorsolateral (top) and dorsomedial (bottom) striatum projected onto coronal sections representing the target coordinates (dorsolateral: $\mathrm{AP}=-0.5 \mathrm{~mm}$; dorsomedial: $\mathrm{AP}=1.7 \mathrm{~mm}$ from bregma); recording sites extended $\sim \pm 0.5 \mathrm{~mm}$ along the anterior-poster axis. $\boldsymbol{B}$, Rats were trained on a T-maze task in which blocks of auditory (top) and tactile (bottom) trials were interleaved within daily sessions. Dashed line indicates location of auditory cue onset. Shading represents zone of tactile cue presentation. C, Percentage correct performance on auditory (dark gray) and tactile (light gray) task versions across training blocks defined according to percentage correct performance on the two versions of the task. Training Block A: sessions in which performance on both versions was $<72.5 \%$ correct (dashed line). Training Block B: sessions in which auditory performance was $>72.5 \%$ but tactile performance remained $<72.5 \%$ correct. Training Block C: sessions in which performance on both versions was $>72.5 \%$ correct. Boxes represent 25 th to 75 th percentiles. Whiskers represent most extreme data points. $\boldsymbol{D}$, Recorded units were classified as putative FSIs (red) or MSNs (blue) by clustering according to wave-shape and firing rate criteria. TANs (green) were not distinguishable using these criteria. $\boldsymbol{E}$, Putative TANs were separated from FSIs and MSNs according to bursting, ISI, and postspike suppression parameters (see Materials and Methods). $\boldsymbol{F}$, Percentages of units classified as MSNs, FSIs, and TANs in dorsolateral (left) and dorsomedial (right) striatum in the population of neurons that included units repeated across multiple sessions. G, Percentages of units classified as MSNs, FSIs, and TANs after removing repeat units. $\boldsymbol{H}$, Percentages of task-responsive units found in dorsolateral (light bars) and dorsomedial (dark bars) striatum for each subtype. Error bars indicate $95 \%$ confidence limits (bootstrap estimate, 1000 bootstraps).

unbiased pairwise phase consistency (PPC) measure of Vinck et al. (2012). We found good agreement between the two methods and report the results of the unbiased PPC method. To determine the percentage of entrained neurons around each task event ( \pm 1 s perievent windows), a PPC threshold was determined from shuffled data. For each single unit and each perievent window, ISIs were shuffled for each trial, so that spike times were shuffled with respect to the LFP, but spiking statistics were preserved within each window. PPC was then calculated for the shuffled data, and the PPC threshold was determined for each event by bootstrapping the PPC results for the shuffled population and taking the 95th percentile of the resulting distribution.

Movement-related analysis of rhythmic bursty FSIs. The correlation between the animal's running speed and the interburst interval of each rhythmically bursting unit was calculated. Interburst interval was calculated by converting the spike train to a continuous ( $1 \mathrm{kHz}$ sampled) waveform by placing a 1 at the sample closest to each spike time, and a 0 at all other points. The signal was then smoothed by applying a 250 -point moving average filter, and burst times were identified as the peaks of the smoothed signal during the time interval between locomotion onset and goal-reaching. Trial duration was computed as the time between gate opening and goal-reaching for each trial, and the Pearson's linear correlation coefficient was computed between the trial durations and median interburst intervals for each trial.

Identification of modality-discriminative, turn-discriminative, and outcome-discriminative interneurons. Modality-discriminative neurons were identified by comparing the mean spike counts within $\pm 300 \mathrm{~ms}$ perievent windows around each task event during auditory versus tactile trials using a standard $t$ test assuming unequal variances. Units were accepted as significantly discriminative if $p<0.05$. Turn-discriminative and outcome-discriminative neurons were identified similarly, for rightversus left-turn trials and for correct versus incorrect trials, respectively. The percentage of neurons preferring each turn direction or trial outcome was then calculated along with the bootstrap 95\% confidence limits (1000 bootstraps).

We additionally tested for significant interaction between entrainment and discrimination of modality, turn, or outcome conditions. Entrainment of each single unit was determined for each task event by calculating the PPC value across all trials during which the unit was recorded. We then used a Fisher's Exact test to determine whether, for each discrimination condition and perievent period, significant interaction occurred, with a Bonferroni corrected significance level of $p=0.0056$.

\section{Results}

For all analyses, individual sessions were grouped into one of three training blocks: early, middle, and late in training, termed Blocks A-C, respectively, based on distinct changes in behavioral performance that we observed during the training. Early in training (Block A), the percentage correct performance achieved on both the auditory and tactile versions of the task remained below the $72.5 \%$ correct learning criterion (Fig. 1C). Only sessions in which the rats completed at least 35 trials were included. All rats reached criterion performance on the auditory version of the task first, and Block B included those sessions in which performance on the auditory version of the task was $>72.5 \%$ correct, but performance on the tactile version of the task remained below criterion. Thus, during Block B, there was a discrepancy between behavioral performance on the two tasks, even though they were both performed on the same days. Finally, Block C consisted of those late-stage training sessions, for the animals in Group 2, in which performance was above criterion on both versions of the task (Thorn et al., 2010).

Of the 6750 manually identified clusters, 1854 met our basic inclusion criteria for spike count, trial count, and LRatio. After unit classification and cross-session matching procedures were performed, 290 well-isolated FSI spike clusters corresponding to 84 nonrepeating units (dorsolateral: $n=31$; dorsomedial: $n=$ 53 ) and 98 TAN spike clusters corresponding to 39 units (dorsolateral: $n=20$; dorsomedial: $n=19$ ) were accepted for further analysis. For MSNs, 1217 spike clusters were accepted, corresponding to 1024 nonrepeating units (dorsolateral: $n=531$; dorsomedial: $n=493$ ). An additional 249 well-isolated clusters had wave shapes or firing rates that fell outside the thresholds set for classification as one of these subtypes, and these "Other" units 

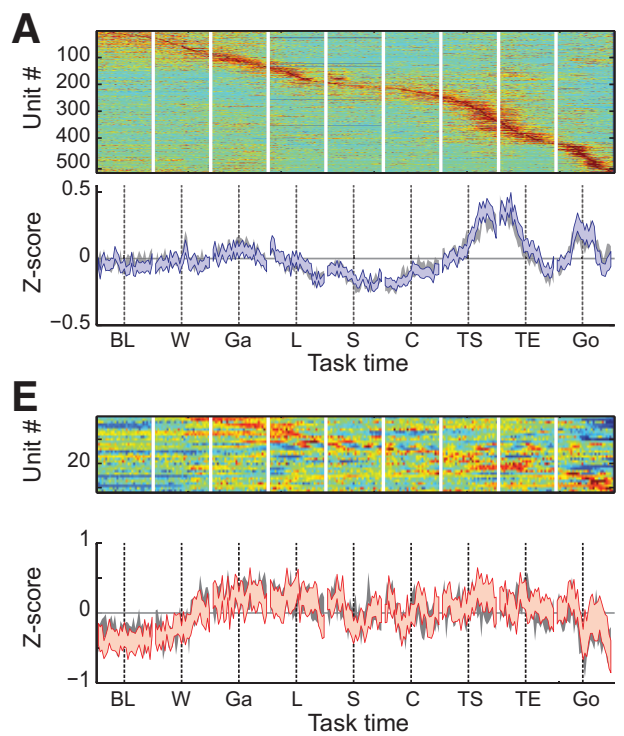

1

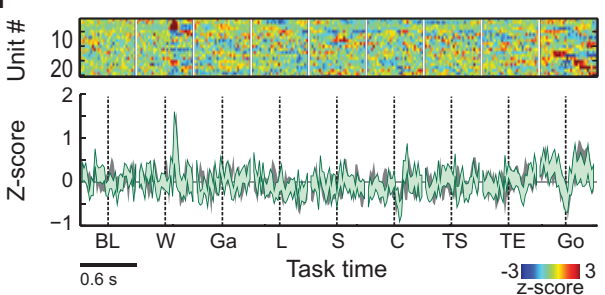

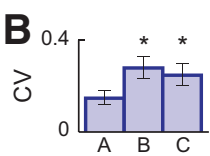
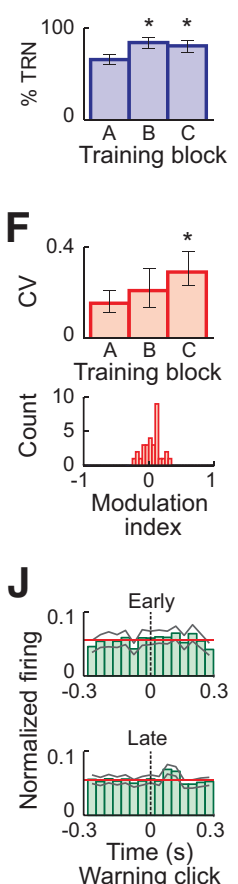
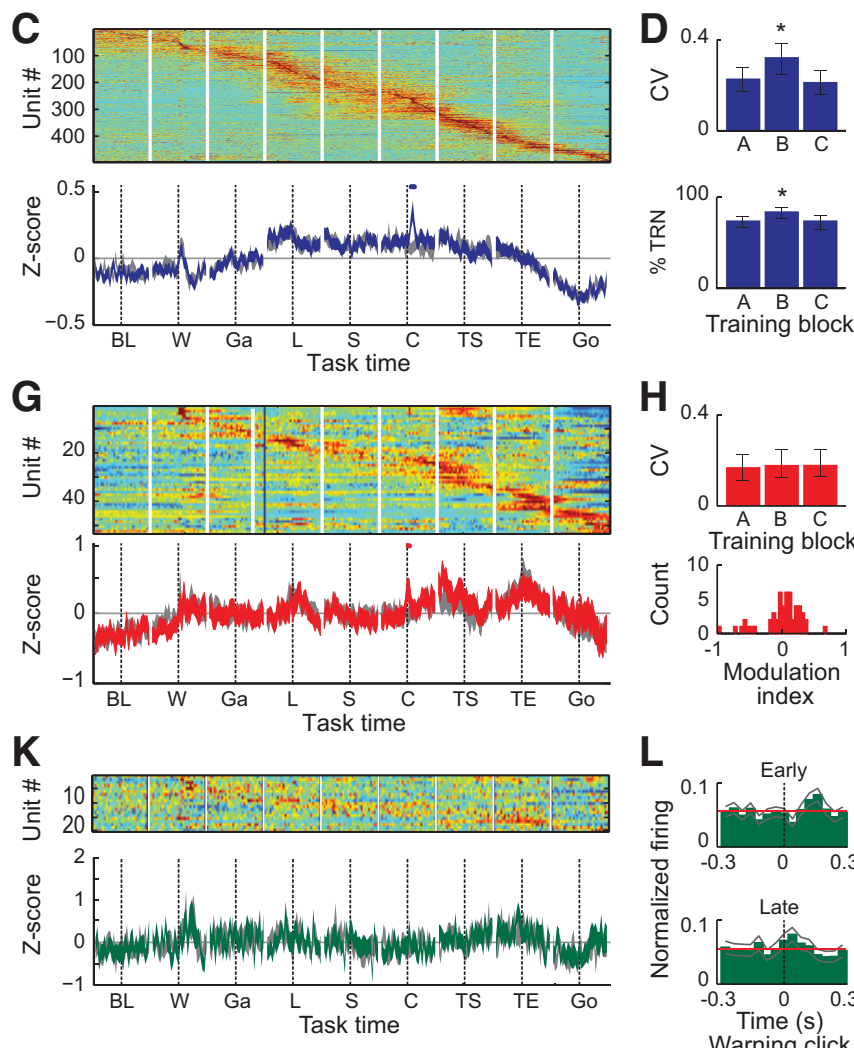

Figure 2. Population activity of MSNs, FSIs, and TANs in dorsolateral and dorsomedial striatum. $\boldsymbol{A}, \boldsymbol{C}$, Session-averaged activity of dorsolateral $(\boldsymbol{A})$ and dorsomedial ( $\boldsymbol{C}$ ) MSNs across task-time. Color plots (top) represent z-score normalized activity for each unit classified as MSN. Line plots (bottom) represent that average activity across all MSNs in each region is nearly identical during auditory (blue) and tactile trials (gray). Shading represents SEM. Dots above line plots indicate bins with significant difference between the two task versions ( $p<0.05$ ). BL, Baseline; $W$, warning click; Ga, gate opening; L, locomotion onset; S, out-of-start; C, cue onset; TS, turn start; TE, turn end; Go, goal-reaching. Scale bars for all plots are shown at bottom left of figure. $B$, D, The CV of population firing across task-time (top) and the percentage of task-responsive units (bottom) for MSNs recorded in the dorsolateral (B) and dorsomedial (D) striatum. Error bars indicate $95 \%$ confidence limits (bootstrap estimate, 1000 bootstraps). ${ }^{*} p<0.05 . \boldsymbol{E}, \boldsymbol{G}$, Same as in $\boldsymbol{A}, \boldsymbol{C}$ for FSls in dorsolateral $(\boldsymbol{E})$ and dorsomedial $(\boldsymbol{G})$ striatum. $\boldsymbol{F}, \boldsymbol{H}, \mathbf{C V}$ across training blocks (top) and MI comparing in-task firing rate to out-of-task firing rate (bottom) for FSIs in dorsolateral $(\boldsymbol{F})$ and dorsomedial $(\boldsymbol{H})$ striatum. $\boldsymbol{I}, \boldsymbol{K}$, Same as in $\boldsymbol{A}, \boldsymbol{C}$ for TANs in dorsolateral $(\boldsymbol{I})$ and dorsomedial $(\boldsymbol{K})$ striatum. $\boldsymbol{J}, \boldsymbol{L}$, TANs in dorsolateral $(\boldsymbol{J})$ and dorsomedial $(\boldsymbol{L})$ striatum strengthen their phasic response to the warning click from Block A ("Early," top) to Blocks B and C ("Late," bottom). Perievent histogram represents population mean activity in 40 ms bins normalized on a $0-1$ (minimum-maximum) scale. Gray lines indicate $95 \%$ confidence limits (bootstrap estimate, 1000 bootstraps). Red lines indicate expectation for a uniform firing distribution across the 15 bin window.

were not analyzed further (Fig. $1 F$ ). Despite the exclusion of a large fraction of the manually identified clusters, we found that, once repeat units were accounted for, $\sim 90 \%$ of accepted units were classified as projection neurons (Fig. $1 G$ ), a value consistent with our previous report, and with known subtype densities within the striatum (Kemp and Powell, 1971). The distribution of interneuron subtypes, however, was found to differ significantly between dorsomedial and dorsolateral striatum $\left(p=0.03, \chi^{2}\right.$ test on neuron populations without repeats) because of the relatively high number of FSIs found medially (Fig. $1 G$ ). For each subtype, equal percentages were classified as "task-responsive" in the two regions (Fig. $1 H$; MSNs: $p=0.942$; FSIs: $p=0.647$; TANs: $p=$ 1.0; Fisher's Exact test on neuron populations without repeats).

Striatal interneuron subtypes exhibit task-specific and training-related modulation of population spike activity

For each cell type, population firing rates were nearly identical during performance of the auditory and tactile versions of the task (Fig. 2). Only during a 40-60 ms window after the onset of the conditional cue mid-task did dorsomedial MSN and FSI populations exhibit different firing rates during the two conditions. Given these minimal differences, we combined all trials for the population analyses below.
Analysis of the neurons classified as MSNs indicated that approximately three-fourths of MSNs in both dorsolateral ( $n=400$ of $531,75.3 \%)$ and dorsomedial $(n=369$ of $492,75.0 \%)$ striatum were task-responsive (Fig. $1 H$ ). This percentage is higher than that reported for earlier T-maze studies (Barnes et al., 2005; Thorn et al., 2010; Smith and Graybiel, 2013), likely because of our exclusion here of low-firing MSNs that are predominantly non-task-responsive but that also correspond to less well-isolated clusters. Despite this difference from our previous report, we nonetheless confirmed that dorsolateral MSN firing emphasizes the action boundaries of the task at start, turn, and goal-reaching (Fig. 2A), whereas population firing mid-task is suppressed. By contrast, dorsomedial MSNs exhibit the highest firing rates midtask (Fig. 2C). We also found, as before, a contrast between the modulations of these firing patterns over the extended training periods of the task. The dorsolateral MSN population activity became increasingly nonuniform throughout training (Fig. $2 B$, top; Blocks B and C: $p<0.001$, bootstrap estimate), and the percentage of dorsolateral MSNs classified as task-responsive (TRN) also increased across training blocks (Fig. 2B, bottom; Block B: $p<0.001$, Block C: $p=0.001$, bootstrap estimate), suggesting that the dorsolateral pattern expression strengthened progressively as the rats acquired the T-maze task. By contrast, 

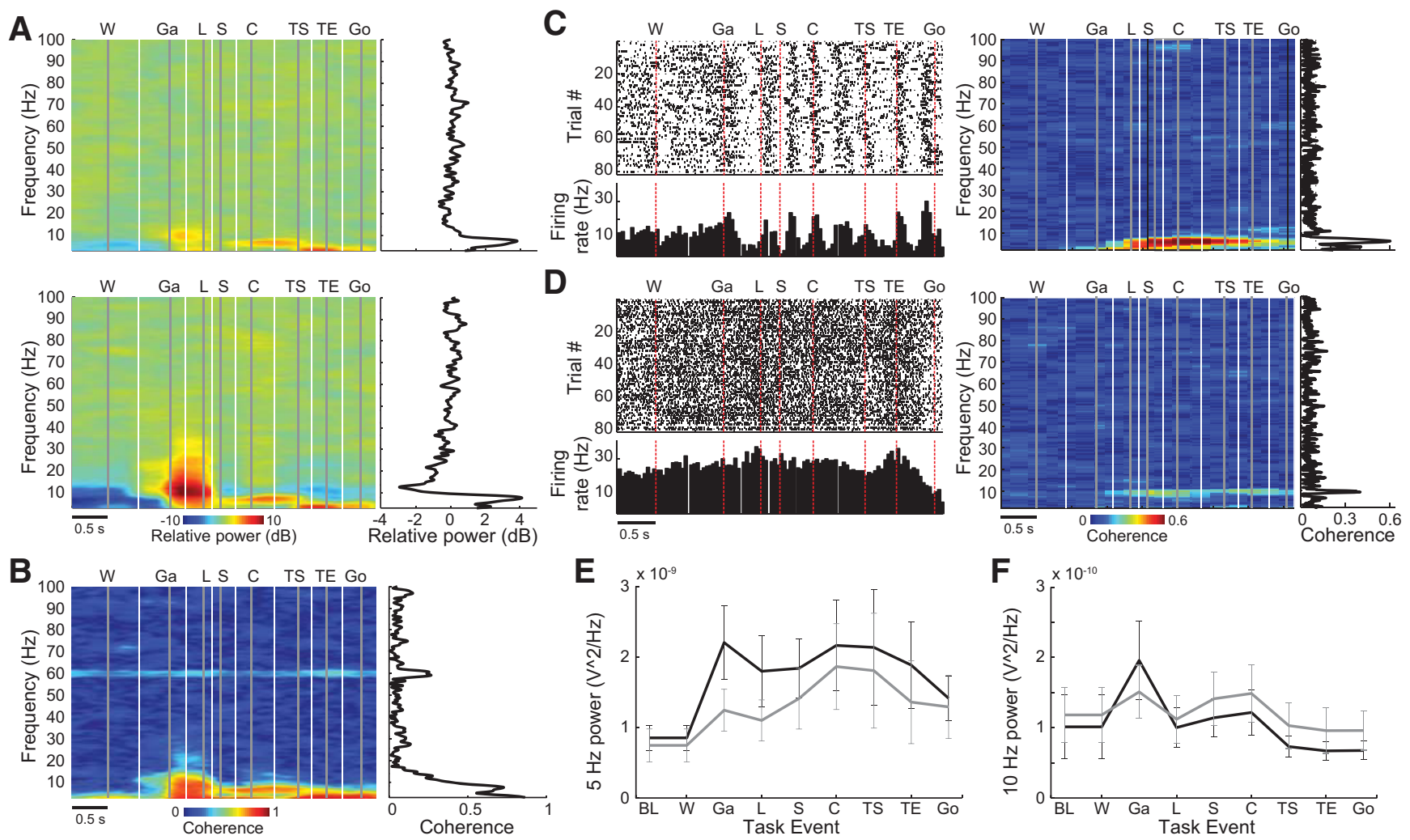

Figure 3. Dorsolateral and dorsomedial striatal recordings exhibit significant LFP power, LFP coherence, and spike-LFP entrainment within the theta band. $A$, Dorsolateral (top) and dorsomedial (bottom) LFPs exhibit similar task-related profiles (left) and average power (right) across a broad frequency range, dominated by theta-band peaks during task performance. Event codes as in Figure 2. $\boldsymbol{B}$, Coherence between LFPs in the two regions peaks mid-task in the theta $(5-12 \mathrm{~Hz}$ ) range. $\boldsymbol{C}, \boldsymbol{D}$, Perievent raster plots and spike histograms across task-time (left) and spike-LFP coherence (right) for single FSIs recorded in dorsolateral $(\boldsymbol{C})$ and dorsomedial $(\boldsymbol{D})$ striatum. $\boldsymbol{E}, \boldsymbol{F}$, LFP power at both low-theta $(5 \mathrm{~Hz}, \boldsymbol{E})$ and high-theta $(10 \mathrm{~Hz}, \boldsymbol{F})$ frequencies exhibit similar dynamics across task performance in dorsolateral (gray) and dorsomedial (black) striatum. Error bars indicate SEM.

patterned activity within the dorsomedial MSN population first became significantly stronger ( $p=0.013$, bootstrap estimate), and the percentage of TRNs was significantly elevated $(p=0.012$, bootstrap estimate) during Block B (Fig. 2D), the period of training during which the animals were performing well on the auditory version of the task, but not on the tactile version. Then, during the final block of training, once good performance had been reached on both versions of the task, the dorsomedial MSN population firing again became more uniform across task-time ( $p=0.311$, bootstrap estimate), and the percentage of TRNs returned to initial levels ( $p=0.465$, bootstrap estimate). These patterns confirm contrasting learning-related dynamics for the putative projection neuron populations in the dorsolateral and dorsomedial striatum.

For the population of units classified as FSIs, nearly all were found to be task-responsive in both the dorsolateral ( $n=30$ of $31,96.8 \%)$ and dorsomedial ( $n=49$ of $53,92.5 \%)$ striatum (Figs. $1 H$ and $2 E, G)$. In the dorsolateral striatum, FSIs showed significantly less uniform firing across task-time late in training (Fig. $2 F$, top; Block C: $p<0.001$, bootstrap estimate), suggesting a strengthening of patterned neural firing for this population across training blocks. FSIs developed stronger firing around task-start and task-end events, and weaker firing mid-task as training progressed, thus exhibiting task-specific and trainingrelated dynamics similar to that of the simultaneously recorded dorsolateral MSNs.

In the dorsomedial striatum, the FSI population exhibited its highest firing mid-task, similar to the MSNs in this region (Fig. $2 G$ ). Unlike the MSN population, we did not observe a significant change in patterned activity across training blocks for dorsomedial FSIs (Fig. $2 H$, top; Block B: $p=0.425$, Block C: $p=0.404$, bootstrap estimate). The FSI population in dorsomedial striatum was, however, notably large and heterogeneous. Among these, eight stood out by virtue of their strong negative modulation indices ( $\mathrm{MI}<-0.4$; Fig. $2 \mathrm{H}$, bottom), indicating that these FSIs greatly reduced their firing in-task, in contrast to the firing of the majority of the FSIs in dorsomedial striatum. Even after the exclusion of this strongly modulated subset, however, no significant changes in the uniformity of FSI firing activity across training blocks were observed (Block B: $p=0.263$; Block C: $p=0.223$, bootstrap estimate).

Among the units identified as TANs, few differences were observed between dorsolateral and dorsomedial populations. Approximately half of the TANs in both regions were found to be task-responsive (Fig. $1 H$; lateral: $n=11$ of 20, 55.0\%; medial: $n=$ 11 of $19,57.9 \%)$. TAN populations in both regions exhibited enhanced phasic activation after the presentation of the warning click denoting trial-start (Fig. $2 I-L$ ), and these phasic responses developed across training sessions (Fig. $2 \mathrm{~J}, L$; dorsolateral: $p=$ 0.003 , dorsomedial: $p<0.001$; bootstrap estimate). TANs in both regions also exhibited reduced activity around the time of goalreaching (Fig. $2 I, K$ ). Only around the onset of the conditional cue mid-task was any region-specific difference observed among the TAN populations. In the dorsolateral striatum, TANs exhibited a brief pause after cue onset, a response that was not observed in the population firing of dorsomedial TANs.

For FSIs, especially in the dorsolateral striatum, we found that population firing dynamics exhibit substantial similari- 
A
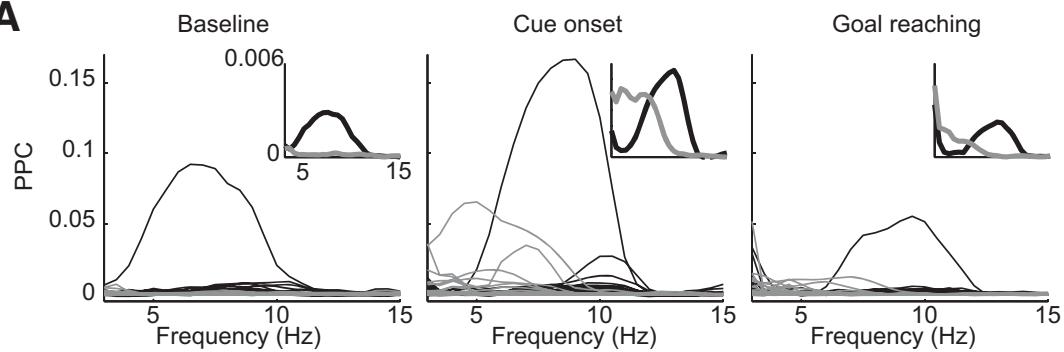

B
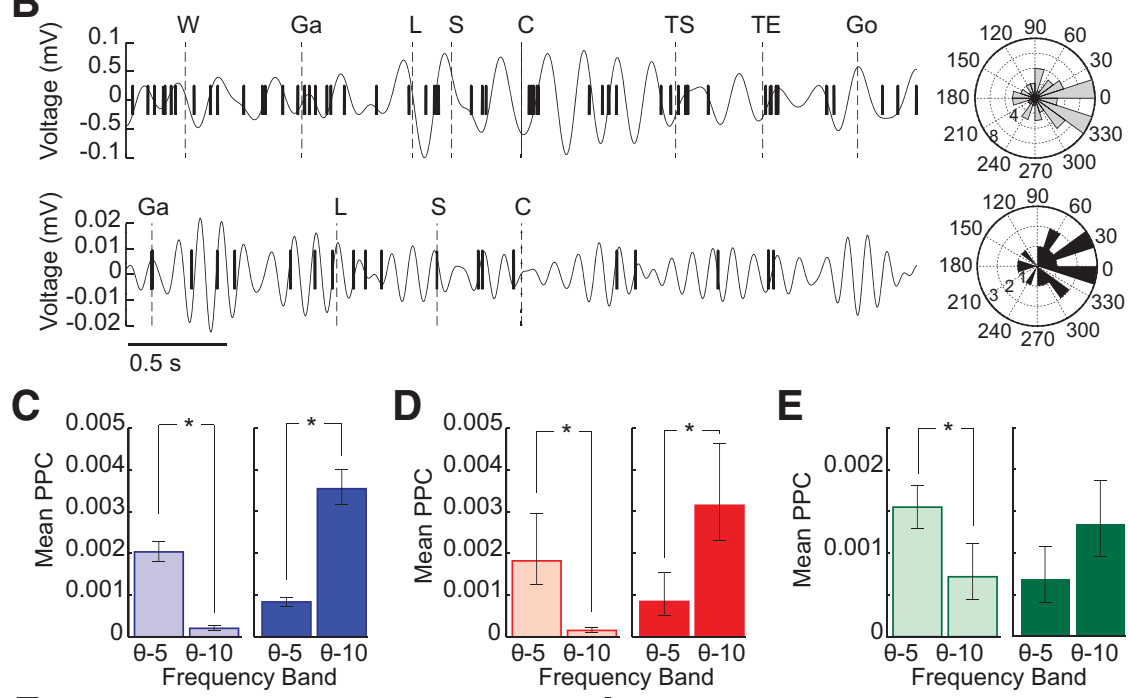

D
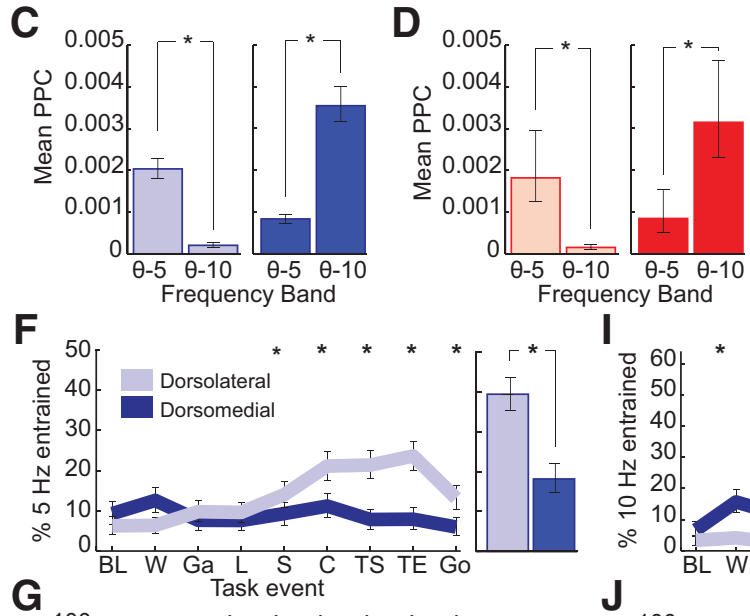

E

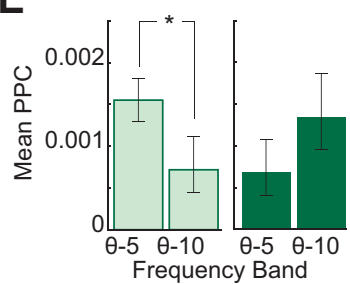

1
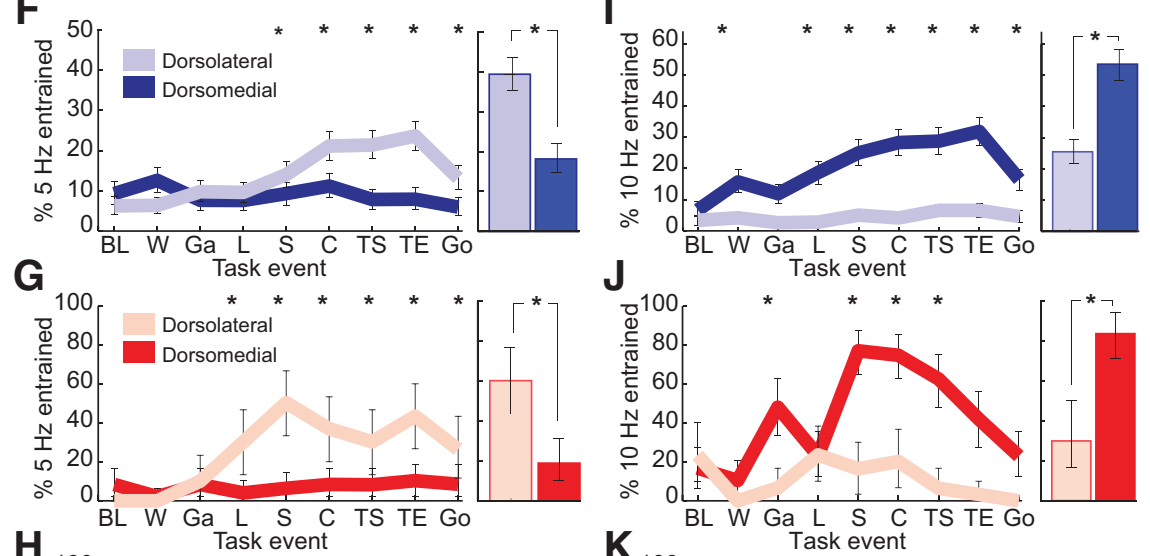

\section{을}
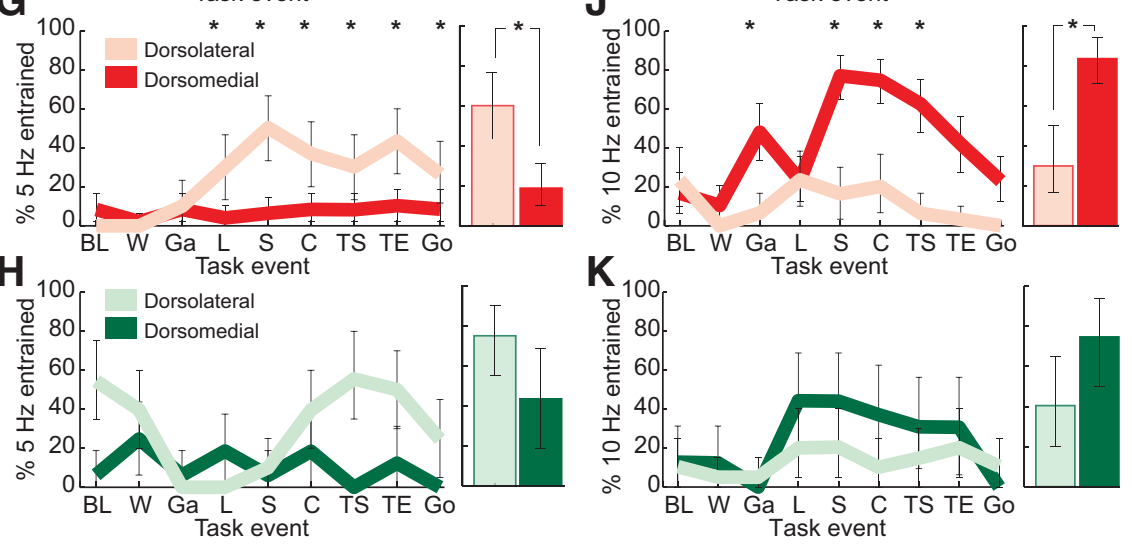

Figure 4. Dorsolateral and dorsomedial striatal subtypes are entrained to different theta-band frequencies. $\boldsymbol{A}, \mathrm{PPC}$ for each recorded FSI in dorsolateral (light gray) and dorsomedial (dark gray) striatum across a range of center frequencies, computed for $2 \mathrm{~s}$ windows centered on baseline, cue onset, and goal-reaching. Insets, Mean PPC for dorsolateral and dorsomedial FSI populations. $\boldsymbol{B}$, Single-unit examples illustrating spike occurrences at a consistent phase of LFP oscillations for a dorsolateral FSI entrained to $\sim 5 \mathrm{~Hz}$ (top) and dorsomedial FSI entrained to $\sim 10 \mathrm{~Hz}$ (bottom). $\boldsymbol{C}-\boldsymbol{E}$, Mean PPC for populations of MSNs (C, blue), FSIs (D, red), and TANs ( $\boldsymbol{E}$, green) in dorsolateral (left) and dorsomedial (right) striatum. Dorsolateral subtypes exhibit stronger low-theta than high-theta entrainment, whereas the opposite is true for dorsomedial subtypes. Error bars indicate bootstrap $95 \%$ confidence intervals (1000 bootstraps). ${ }^{*} p<0.05 . \boldsymbol{F}-\boldsymbol{H}$, Percentage of MSNs $(\boldsymbol{F})$, FSIs $(\boldsymbol{G})$, and TANs $(\boldsymbol{H})$ entrained to low-theta $(5 \mathrm{~Hz})$ oscillations across nine different task events (left) and during one or more event intervals from locomotion onset to turn end (right). Error bars indicate bootstrap 95\% confidence intervals (1000 bootstraps). *Significant increase $(p<0.05)$ above baseline percentages entrained. Event codes as in Figure 2. $\boldsymbol{I}-\boldsymbol{K}$, Same as $\boldsymbol{F}-\boldsymbol{H}$ for MSNs $(\boldsymbol{I})$, FSIs $(\boldsymbol{J})$, and TANs $(\boldsymbol{K})$ entrained to high-theta (10 $\mathrm{Hz}$ ) oscillations. $\mathbf{C}-\boldsymbol{K}$, For all plots, lighter shading represents results for dorsolateral subtypes and darker shading represents results for dorsomedial subtypes. ties to the simultaneously recorded MSNs, a result that suggests that shared excitatory inputs rather than local inhibitory processing dominate the shaping of ensemble activity patterns for these subtypes. TANs also did not predict readily the region-specific firing patterns of the MSNs, as ensemble activity for this interneuron population was nearly identical in dorsolateral and dorsomedial striatum. Thus, neither FSIs nor TANs fired in patterns that clearly accounted for the population activity of the MSNs in the corresponding regions of the striatum, even though both sets of putative interneurons did, like the MSNs, exhibit task-related dynamics and learning-related plasticity during $\mathrm{T}$-maze acquisition.

Interneurons and projection neurons are entrained to different theta-band frequencies in the dorsolateral and dorsomedial striatum

We next looked for further evidence of region-specific influences from synaptic activity on striatal processing by analyzing the relationship of single-unit spiking to the ongoing LFP for each neuronal subtype. Consistent with previous studies (DeCoteau et al., 2007b; Berke, 2009), the LFPs in the dorsolateral and dorsomedial striatum were remarkably similar across a broad range of frequencies (Fig. 3A). Coherence between the raw dorsolateral and dorsomedial LFPs was found in the gamma, beta, and theta bands (data not shown). However, this coherence was strongest and most consistent across animals in the theta band $(5-12 \mathrm{~Hz})$. The measured theta-band power remained strong after we subtracted the average LFP recorded across tetrodes in each region to control for volume conduction and reference channel activity (Fig. $3 A$ ), and the theta-band coherence between dorsolateral and dorsomedial LFPs remained high when sampled for these hyperlocal field potentials (Fig. 3B). SpikeLFP coherence was likewise most pronounced for frequencies in the 5-12 $\mathrm{Hz}$ range (Fig. $3 C, D$ ). We therefore focused on the theta band for further analysis of spike-LFP coherence.

Our initial observations suggested that some FSIs exhibited robust rhythmic firing at $\sim 5 \mathrm{~Hz}$ and that these were strongly coherent with the ongoing LFP at the same low-theta frequency (Fig. 3C). This type of rhythmic firing was apparent in the perievent raster and histogram plots of several $(n=6$ of $31,19.4 \%$ ) dorsolateral FSIs, but such clear rhythmic activity was 
never observed among dorsomedial FSIs. Nonetheless, dorsomedial FSIs often exhibited significant theta-band coherence with the LFPs, but at a slightly higher frequency centered at $\sim 10 \mathrm{~Hz}$ (Fig. 3D). This region-specific dichotomy in theta-band spikeLFP entrainment was not reflected in the raw LFP power in either the low-theta or high-theta bands; LFP power did not differ between dorsolateral and dorsomedial regions for either $5 \mathrm{~Hz}$ or 10 $\mathrm{Hz}$ center frequencies (Fig. $3 E, F$ ).

Based on our preliminary observations, we next investigated the possibility that local striatal networks might be entrained systematically to different frequencies by testing the PPC (Vinck et al., 2012) for each unit after filtering the LFP at different center frequencies in the $3-15 \mathrm{~Hz}$ range. Confirming our initial impression, the mean frequency of strongest entrainment was $\sim 5 \mathrm{~Hz}$ for all dorsolateral neuron subtypes, whereas dorsomedial subtypes were best entrained at $\sim 10 \mathrm{~Hz}$. This pattern is illustrated for FSIs in Figure $4 A, B$; we found similar preferential entrainment for all neuron subtypes (Fig. $4 C-E)$.

To quantify the changes in entrainment across task-time, we measured the percentage of the striatal projection neurons and interneurons significantly entrained $(\mathrm{PPC}>95$ th percentile threshold of shuffled data) to $5 \mathrm{~Hz}$ and $10 \mathrm{~Hz}$ oscillations in the LFPs during a $\pm 1 \mathrm{~s}$ window around each task event. In the dorsolateral striatum, the proportion of MSNs and FSIs entrained to $5 \mathrm{~Hz}$ oscillations increased in-task compared with the baseline period, with the largest number of entrained units found between out-of-start and turn end events (Fig. 4F, G, left). More MSNs and FSIs were entrained to low-theta oscillations during this period in the dorsolateral striatum than in the dorsomedial striatum, with $\sim 40 \%$ of dorsolateral MSNs (198 of 501, 39.5\%) and $60 \%$ of dorsolateral FSIs (18 of 30,60.0\%) exhibiting entrainment to $5 \mathrm{~Hz}$ rhythms during one or more of these in-task events (Fig. 4F, G, right). TANs showed a similar trend for dorsolateral rather than dorsomedial units to be entrained preferentially to low-theta oscillations (Fig. $4 H$ ), but this difference failed to reach statistical significance. Dorsolateral entrainment of MSNs, FSIs, and TANs to the higher-frequency $10 \mathrm{~Hz}$ oscillations in the LFP remained constant at low levels throughout task-time (Fig. $4 I-K$ ).

In contrast to these dorsolateral striatal neurons, dorsomedial subtypes were preferentially entrained to $10 \mathrm{~Hz}$ rhythms rather than to $5 \mathrm{~Hz}$ rhythms. Increasing numbers of dorsomedial MSNs and FSIs became entrained to high-theta oscillations from baseline to mid-task events (Fig. 4I,J, left). Using the same period from out-ofstart to turn-end that was used above, more than half of dorsomedial MSNs (225 of $420,53.6 \%$ ) and $83.3 \%$ of dorsomedial FSIs ( 40 of 48 ) were entrained during one or more of these mid-task events (Fig. $4 I, J$, right). For the TANs, these distinctions were less clear; dorsomedial TANs exhibited a slight preference for higher-frequency theta-band entrainment compared with dorsolateral TANs, but this relationship failed to reach significance around any task event, or across all mid-task events combined (Fig. $4 K$ ). Entrainment of dorsomedial neurons of all subtypes to the lower-frequency $5 \mathrm{~Hz}$ rhythms remained low throughout task-time (Fig. $4 F-H$ ).

Collectively, these analyses demonstrate that entrainment of dorsolateral and dorsomedial neurons to theta-band rhythms is dynamically modulated during task performance, with the firing of neuronal subtypes in both regions becoming synchronized to the LFP during the same mid-task period. Theta-band entrainment of spikes in these two regions is distinguished, however, by their simultaneous entrainment to different specific frequencies within the theta-band.
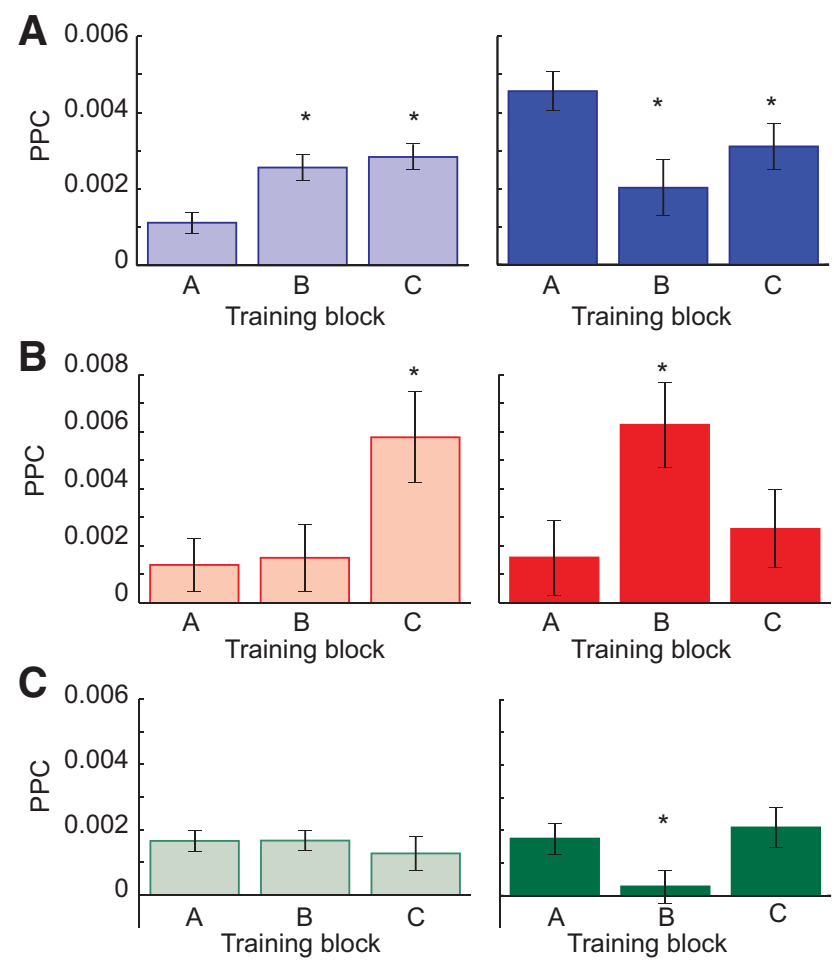

Figure 5. Strength of spike-LFP entrainment in dorsolateral and dorsomedial striatum to preferred theta-band rhythms is modulated across training blocks. $A$, Mean PPC and bootstrap 95\% confidence intervals for MSN populations in dorsolateral (left, light blue) and dorsomedial (right, dark blue) striatum. ${ }^{*} p<0.05$, relative to Block A. B, C, Same as in $\boldsymbol{A}$ for populations of FSIs $(\boldsymbol{B})$ and TANs (C).

\section{Entrainment of striatal subtypes to theta-band rhythms is dynamically modulated across learning}

We next asked whether the strength of entrainment of striatal subpopulations also varied across training blocks. In both regions, we found learning-related dynamics in spike-LFP synchronization that paralleled other changes in population activity.

Dorsolateral MSNs exhibited significantly higher PPC in Training Blocks B and C compared with Training Block A, indicating that they became increasingly entrained to low-theta rhythms as training progressed (Fig. 5A, left). The development of MSN entrainment in the dorsolateral striatum closely coincided with the development of patterned activity in this region and the increase in task-responsive MSNs across training blocks (Fig. 2B). Similarly, dorsolateral FSIs showed significantly stronger entrainment during Block $C$ late in training compared with the initial training block (Fig. 5B, left), dynamics that again coincide with the emergence of patterned activity for this population (Fig. $2 F$ ). For dorsolateral TANs, mean PPC values were consistently low throughout training, and we did not observe any significant learning-related changes for this small population (Fig. $5 C$, left).

In the dorsomedial striatum, neurons likewise exhibited dynamics in high-theta entrainment across training blocks, but the direction of these changes lacked consistency across subtypes. MSNs had a significant reduction in PPC from Block A to Block $\mathrm{B}$, and PPC remained low during Training Block C (Fig. 5A, right), indicating that the strength of entrainment of dorsomedial MSNs to high-theta rhythms declines as performance improves on the T-maze task. FSIs in dorsomedial striatum showed significantly higher PPC values during the middle training block, but these returned to initial low levels late in training (Fig. $5 C$, right). 
A
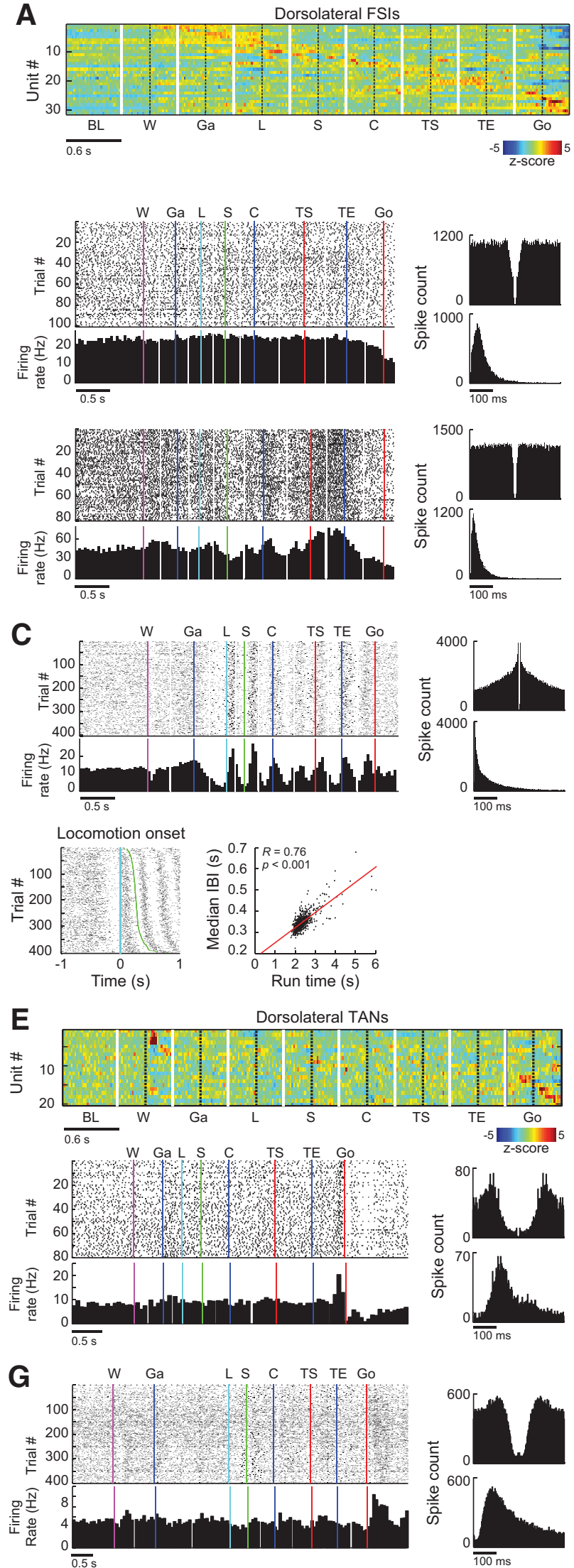
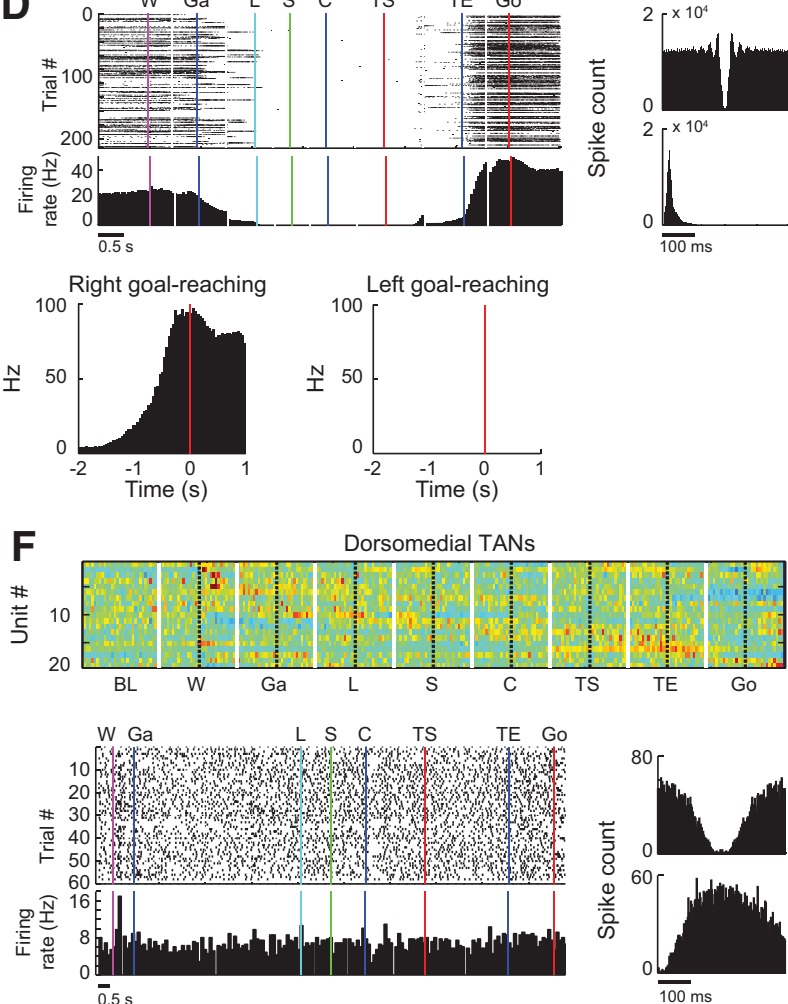

H
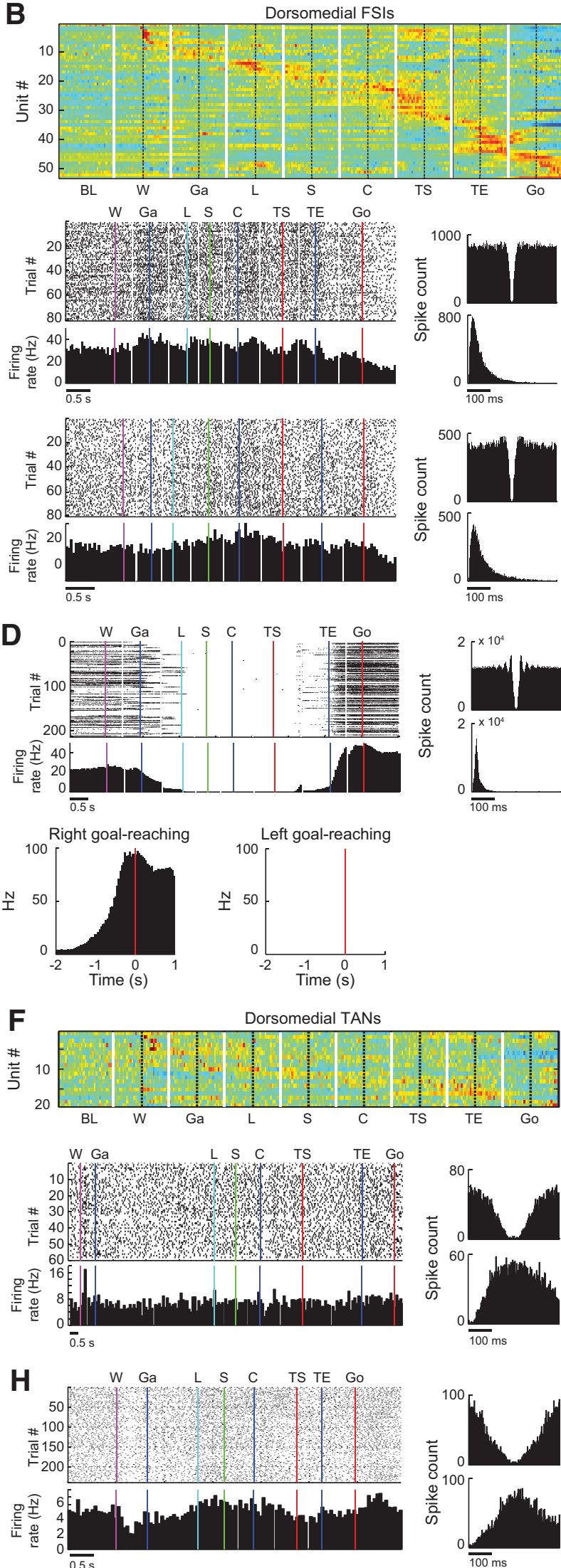

Figure 6. FSIs and TANs exhibit a variety of task-related activities. $\boldsymbol{A}, \boldsymbol{B}, \mathbf{Z}$-score normalized, session-averaged activity for each FSI recorded in dorsolateral $(\boldsymbol{A})$ and dorsomedial ( $\boldsymbol{B})$ striatum, sorted according to the time of maximum firing in-task (top) and perievent raster plots and histograms illustrating examples of task-related firing of FSIs (bottom) with corresponding autocorrelogram (top right) and ISI histogram (bottom right) for each FSI. Color and time scales for both heat plots shown in $\boldsymbol{A}$. Event abbreviations as in Figure 2. C, Top, Task-related activity of a distinctive "rhythmic bursty" FSI recorded in dorsolateral striatum across several sessions. Bottom, Perievent raster plot centered on locomotion onset (time 0) with trials reordered (Figure legend continues.) 
Finally, the small population of TANs recorded in the dorsomedial striatum, like those in the dorsolateral striatum, was not strongly entrained to ongoing theta-band rhythms during any training block. Nonetheless, we did find a significant dip in entrainment strength during mid-training Block B for this subtype. Overall, spike-LFP entrainment in the dorsomedial striatum was strongest in the early (for MSNs) and middle (for FSIs) training blocks, with entrainment generally declining by the late-training period. By contrast, in the dorsolateral striatum entrainment increased throughout learning and was highest in the latest stages of training, once entrainment among dorsomedial striatal neurons had waned.

To investigate the possibility that the dynamics we observed in entrainment of striatal neurons to ongoing theta-band rhythms could be related to changes in the motor performance of the animals across task-time and training, we examined the effect of running speed on PPC for all subtypes. Running speeds tend to increase across task-time and training blocks, with trends that generally tracked the changes in entrainment strength that we observed, particularly for neuronal subtypes in the dorsolateral (sensorimotor) striatum. To determine whether neurons exhibited stronger spike-LFP entrainment when the animals were running faster in the maze, we divided trials in each session into quartiles based on the animal's running time from out-of-start to goal-reaching events. We then measured PPC during this task period for trials in quartile 1 (fastest trials) and quartile 4 (slowest trials) and asked whether a significant difference in entrainment existed between quartiles for any subtype in either region. We found no significant difference in the strength of entrainment mid-task during slower trials and faster trials; this was true for all subtypes in both regions (dorsolateral MSNs: $p=0.39$, dorsomedial MSNs: $p=0.58$, dorsolateral FSIs: $p=0.52$, dorsomedial FSIs: $p=0.26$, dorsolateral TANs: $p=0.088$, dorsomedial TANs: $p=0.89$; Wilcoxon signed-rank test). Thus, although theta-band entrainment was strongest during periods of locomotion, and strengthened for dorsolateral subtypes as animals improved their motor performance on the maze, running speed per se was not a primary driver of theta-band entrainment in either region.

We also tested whether PPC values were consistent across stimulus, action and outcome conditions in our task. We found that none of these task parameters were associated with differences in single-unit entrainment to theta-band LFP rhythms for any subtype in either region. PPC values did not differ significantly between auditory and tactile stimulus conditions for any subtypes (dorsolateral MSNs: $p=0.225$, dorsomedial MSNs: $p=$ 0.404 , dorsolateral FSIs: $p=0.600$, dorsomedial FSIs: $p=0.918$, dorsolateral TANs: $p=0.204$, dorsomedial TANs: $p=0.877$; Wilcoxon signed-rank test). Nor was the performed right or left turn action associated with entrainment strength for any subtype (dorsolateral MSNs: $p=0.254$, dorsomedial MSNs: $p=0.290$, dorsolateral FSIs: $p=0.329$, dorsomedial FSIs: $p=0.383$, dorsolateral TANs: $p=0.970$, dorsomedial TANs: $p=0.0787$; Wil-

\section{$\leftarrow$}

(Figure legend continued.) according to the interevent time from locomotion onset to out-ofstart events (left). Interburst interval (IBI) is strongly correlated with running time for this neuron phenotype (right). D, Top, As in C for a distinctive "task-bracketing" FSI found in the dorsomedial striatum. Bottom, Perievent histograms aligned on goal-reaching (time 0 ), illustrating that this $\mathrm{FSI}$ fires strongly as the animal approaches the right goal but remains silent for left goal approach. $\boldsymbol{E}, \boldsymbol{F}$, As in $\boldsymbol{A}, \boldsymbol{B}$ for TANs recorded in dorsolateral $(\boldsymbol{E})$ and dorsomedial $(\boldsymbol{F})$ striatum. $\mathbf{G}$, Task-related activity of a dorsolateral TAN that developed pause-rebound response to warning click and phasic activity after goal-reaching over several training sessions. $\boldsymbol{H}, \mathrm{A}$ dorsomedial TAN that exhibited longer activity pauses after the warning click. coxon signed-rank test). Outcome condition likewise failed to influence PPC values (dorsolateral MSNs: $p=0.217$, dorsomedial MSNs: $p=0.927$, dorsolateral FSIs: $p=0.318$, dorsomedial FSIs: $p=0.566$, dorsolateral TANs: $p=0.067$, dorsomedial TANs: $p=0.278$; Wilcoxon signed-rank test). Thus, entrainment of MSNs, FSIs, and TANs in the dorsolateral and dorsomedial striatum to $5 \mathrm{~Hz}$ and $10 \mathrm{~Hz}$ rhythms, respectively, appears to be a global property of task acquisition and performance that is not tied to specific stimulus, movement, or outcome parameters.

\section{Striatal interneurons exhibit diverse rhythmic and task-related activity}

In light of the striking difference between the dorsolateral and dorsomedial striatum in their patterns of respective spike entrainment to $\sim 5 \mathrm{~Hz}$ and $\sim 10 \mathrm{~Hz}$ theta-band rhythms, we asked whether the detailed characteristics of the interneurons offered clues to how these region-specific population dynamics might emerge.

For the FSIs, we observed diverse task-related responses in both striatal regions; FSIs exhibiting strongest firing at beginning, middle, and end task events were found in both dorsolateral (Fig. $6 \mathrm{~A}$ ) and dorsomedial (Fig. 6B) striatum. However, we detected two highly distinct firing patterns among the FSIs recorded, and these were found to be region-specific (Fig. 6C,D). The first of these FSI subtypes exhibited a "rhythmic bursty" firing pattern and was restricted to the dorsolateral striatum ( $n=6$ of 31 , $19.4 \%$, Figs. $3 C$ and $6 C$ ). These FSIs were unique in exhibiting strongly rhythmic firing mid-task that was clearly observable in the perievent raster plots and histograms of task activity. The rhythmic bursts occurred at $\sim 2.5-5 \mathrm{~Hz}$, and their spikes were significantly coherent with the ongoing LFPs at this low-theta frequency (Fig. $3 C$ ). Firing of these FSIs switched from a tonic, high firing mode to a rhythmic firing mode around locomotion onset, and this rhythmicity terminated at goal-reaching. Both the occurrence of the bursting activity during running and the lowfrequency periodicity of the bursting itself suggested that the rhythmicity of these FSIs could be locomotion related. Indeed, for all 6 FSIs of this subtype, the duration of the interburst intervals was strongly correlated with trial duration, with faster running speeds associated with higher-frequency bursting (Fig. 6C, bottom). As true for the MSNs examined in the quartile analysis, these rhythmically bursting dorsolateral FSIs were strongly entrained within the $5 \pm 2.5 \mathrm{~Hz}$ band during both fast-run and slow-run trials, but they nevertheless exhibited shifts in bursting frequency within this band that were highly correlated with the simultaneously measured running speed.

In the dorsomedial striatum, another unique FSI firing pattern was observed. A subset of FSIs in this region $(n=8$ of 53 , $15.1 \%)$ exhibited an extreme "task-bracketing" pattern of firing: significantly reducing, or even silencing, their firing mid-task, and then firing at high rates as the goal was approached (Fig. $6 D$, top). These unique FSIs corresponded to those exhibiting the strongest negative modulation indices of the FSI population as a whole (Fig. $2 \mathrm{H}$ ), and their pattern of firing was opposite to the firing of the main dorsomedial FSI (and MSN) populations, which fired most strongly mid-task. A particularly striking feature of this FSI population was the extreme direction selectivity observable in their activity at turning. Almost all ( $n=7$ of 8 ) of these "task-bracketing" neurons were significantly turn-discriminative, with several exhibiting almost no spiking during turns to their nonpreferred direction (Fig. $6 D$, bottom). Despite long periods of very low firing rates, we found that the majority of these dorsomedial FSIs simultaneously exhibited turn-discri- 

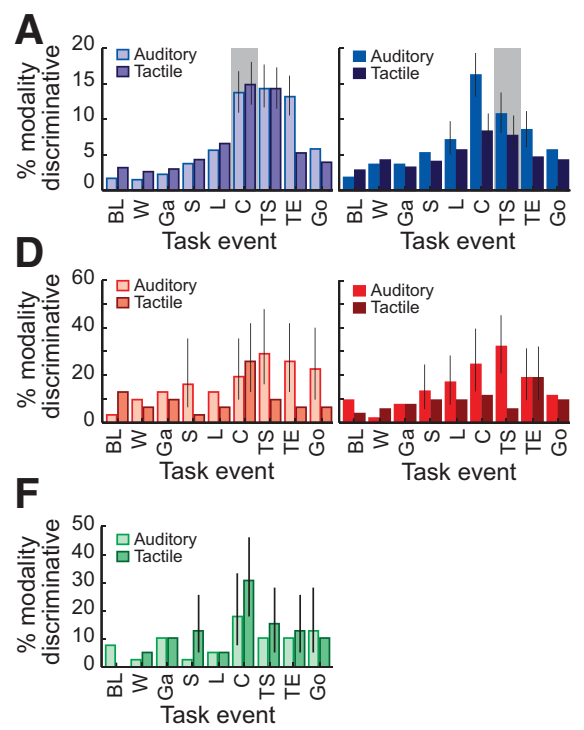

B
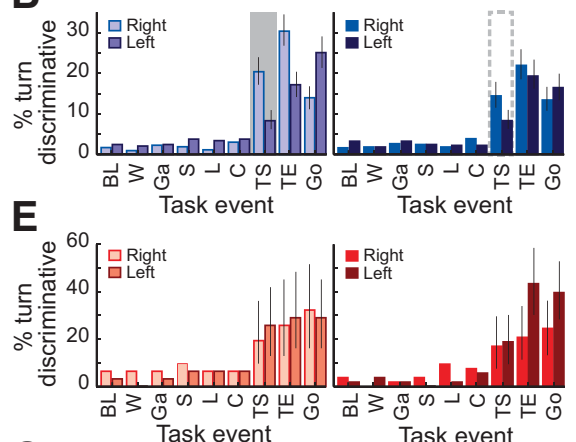

G

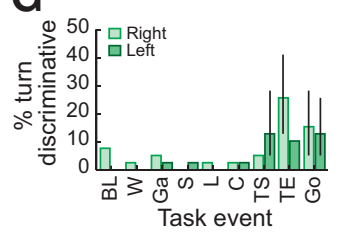

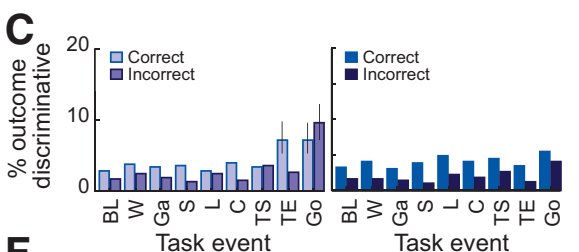

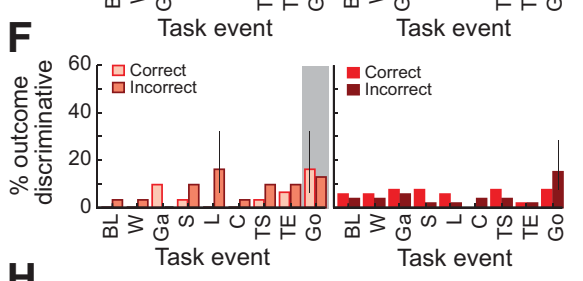

H

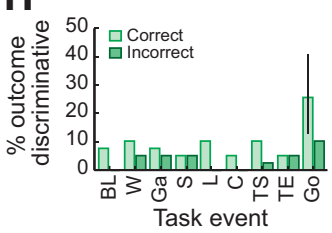

Figure 7. Subpopulations of neurons in dorsolateral and dorsomedial striatum show sensitivity to cue, action, and outcome task parameters. $\boldsymbol{A}, \boldsymbol{D}$, Percentage of MSNs $(\boldsymbol{A})$ and FSIs ( $\boldsymbol{D})$ in dorsolateral (left) and dorsomedial (right) striatum that showed significantly higher firing rates during trials in which auditory (light shading) or during tactile (dark shading) cues were presented. $\boldsymbol{B}, \boldsymbol{E}$, As in $\boldsymbol{A}, \boldsymbol{D}$ for MSNs $(\boldsymbol{B})$ and FSIs $(\boldsymbol{E})$ exhibiting higher firing rates during trials in which right turns (light shading) or left turns (dark shading) were performed. $\boldsymbol{C}, \boldsymbol{F}$, As in $\boldsymbol{A}, \boldsymbol{D}$ for MSNs ( $\boldsymbol{C}$ ) and FSIs (F) exhibiting higher firing during rewarded (light shading) or unrewarded (dark shading) trials. $\boldsymbol{F}-\boldsymbol{H}$, As in $\boldsymbol{A}-\boldsymbol{C}$ for all dorsolateral and dorsomedial TANs combined. For all panels, error bars indicate $95 \%$ bootstrap confidence limits (1000 bootstraps) and are present only for events for which the percentage of discriminative neurons was significantly greater ( $p<0.05$ ) than both the $5 \%$ level expected by chance and the results obtained for shuffled data. Gray shading represents task events during which a significant interaction ( $p<0.0056$, Fisher's Exact test) was found between differential firing and theta-band entrainment. $\boldsymbol{B}$, Dashed gray box indicates task event during which such an interaction was a nonsignificant trend ( $p=0.035$ ).

minative activity and entrainment to the LFP at $\sim 10 \mathrm{~Hz}$ during the turn-goal period ( 5 of 6 for which simultaneously recorded LFPs existed). Notably, although some other FSIs in both dorsolateral and dorsomedial striatum show reduced firing in-task, only these few dorsomedial FSIs showed this remarkable silencing during task performance, setting them apart from all other FSIs recorded in either region.

Closer inspection of the TAN populations in the dorsolateral $(n=19)$ and dorsomedial $(n=20)$ striatum failed to demonstrate region-specific differences in the types of responses exhibited by this subtype (Fig. 6E,F). However, TANs in both regions did exhibit prominent task-related activity, and we combined the dorsolateral and dorsomedial populations for further analyses, in which we tested each TAN for phasic activity around warning click and goal-reaching events. Most TANs in both regions exhibited strong phasic responses around one or both of these task events (Fig. 6E, F; warning click, $n=7$; goal-reaching, $n=8$; both, $n=18$ ). Around warning click, we found TANs that exhibited a single phasic burst $(n=8)$, burst-pause $(n=2)$, burstpause-rebound $(n=3)$, pause only $(n=9)$, and pause-rebound $(n=3)$ types of responses, all typical and well-characterized responses for this neuron type (Aosaki et al., 1995; Morris et al., 2004; Goldberg and Reynolds, 2011). These phasic TAN activities developed and strengthened with training, as was observable both at the population level (Fig. $2 J, L$ ) and in the firing activity of a single dorsolateral TAN that we were able to track across several early training sessions (Fig. $6 G ; p=0.002$, bootstrap estimate). Click-evoked pauses were typically brief, although a small number of TANs instead reduced their firing rates for long periods after the presentation of the warning click (Fig. $6 H ; n=4$ of 39 , $10.2 \%)$. Firing rates for these TANs returned to baseline levels or to levels elevated slightly above baseline, after locomotion onset, which for the sessions in which these TANs were recorded occurred up to $4 \mathrm{~s}$ after warning click presentation. TANs also exhibited phasic responses after the onset of the conditional cue mid-trial ( $n=13$ of $39,33.3 \%$ ), although these were usually weaker in amplitude and shorter in duration than the responses to the warning click by the same neurons (Fig. $6 E-H$ ).

\section{Striatal neurons are more likely to be entrained during periods of task parameter encoding}

To determine how these task-related responses and entrainment to LFP oscillations might contribute to performance of the T-maze task, we asked whether the responses of single MSNs, FSIs, and TANs discriminated between different cue modalities presented, different turn directions performed, or different reward outcomes achieved, and whether the entrainment of single units to their region-specific theta band frequency was related to the encoding of these key task parameters.

For the projection neuron population, we were able to confirm the results of our previous work (Fig. 7A-C) (Thorn et al., 2010). We again found that many dorsolateral and dorsomedial MSNs (dorsolateral: $n=273$ of 531, 51.4\%; dorsomedial: $n=$ 216 of 493, 43.8\%) showed significantly different firing rates during auditory trials compared with tactile trials. The majority of modality-discriminative neurons were found after the onset of the conditional cue (Fig. 7A), although the blocked presentation of the auditory and tactile task-versions made it possible to predict the upcoming stimulus modality and some differential firing was found among MSNs during this precue period. We also found, as before, that a majority of MSNs in both regions (dorsolateral: $n=356$ of 531, $67.0 \%$; dorsomedial: $n=281$ of 493 , $57.0 \%$ ) exhibited differential firing rates during trials containing right versus left turns, but only around trial events after the onset of turning (Fig. $7 B$ ). Few MSNs were sensitive to trial outcome during correct versus incorrect trials (dorsolateral: $n=89$ of 531, $16.8 \%$; dorsomedial: $n=47$ of $493,9.5 \%$ ), and those that were outcome discriminative displayed differential firing rates at the end of the trial, when the animals presumably could sense the presence or absence of reward in the food well (Fig. 7C). 
For the current study, we wished to extend these findings and ask whether striatal neurons that were sensitive to these key parameters (modality, turn direction, and trial outcome) were more likely to be entrained to region-specific theta-band oscillations during task performance. We found that MSNs that were sensitive to cue modality were significantly more likely than nondiscriminative MSNs to be entrained, but only during the task period immediately after cue onset. In the dorsolateral striatum, entrainment to $5 \mathrm{~Hz}$ oscillations by this modality-sensitive population occurred only around the cue onset event (Fig. 7A, left; $p=0.0003$, Fisher's Exact test); during all other task events, modality sensitivity was found to be independent of entrainment ( $p>0.05$, Fisher's Exact test). In the dorsomedial striatum, we detected significant interaction between modality sensitivity and entrainment to the ongoing $10 \mathrm{~Hz}$ rhythms only around the turn start event (Fig. 7A, right; TS: $p=0.004$, other events: $p>0.05$, Fisher's Exact test). For MSNs, only around the turn start event were turn-discriminative MSNs more likely to be entrained than nondiscriminative MSNs; this was true for dorsolateral MSNs (Fig. $7 B$, left; TS: $p=0.002$, other events: $p>0.05$, Fisher's Exact test) and a strong trend for dorsomedial MSNs (Fig. $7 B$, right; TS: $p=0.035$, other events: $p>0.05$, Fisher's Exact test). No significant interaction between outcome sensitivity and entrainment was observed for the small number of MSNs that showed this type of discrimination (Fig. 7C; dorsolateral, Go: $p=0.89$ ). This pattern of results suggests that entrainment of modality-sensitive and response-discriminative projection neurons in the dorsolateral and dorsomedial striatum to $5 \mathrm{~Hz}$ and $10 \mathrm{~Hz}$ rhythms, respectively, is enhanced during a short time window during which these task parameters become immediately relevant to task performance: during trials of both the preferred and nonpreferred condition.

For FSIs, we found activity reflecting sensitivity to modality, turn, and outcome parameters with patterns similar to those seen for MSNs (Fig. 7D-F). A significant proportion of FSIs in both the dorsolateral and the dorsomedial striatum (dorsolateral: $n=$ 22 of 31, 71.0\%; dorsomedial: $n=35$ of $53,66.0 \%$ ) showed differential firing rates during trials in which auditory or tactile cues were presented (Fig. 7D). The majority of discriminative responses were found after the onset of the cue mid-task, but a few FSIs also discriminated cue modality before cue onset. The vast majority of FSIs were sensitive to turn direction, and like MSNs, turn discrimination occurred after the onset of turning (Fig. 7E; dorsolateral: $n=28$ of $31,90.3 \%$; dorsomedial: $n=43$ of 53, 81.1\%). Small percentages of FSIs in both regions discriminated between correct and incorrect trial outcomes (dorsolateral: $n=9$ of $31,29.0 \%$; dorsomedial: $n=12$ of $53,22.6 \%$ ), and these were generally found around goal-reaching. Among modalitysensitive and turn-discriminative FSIs, we did not observe any interaction between differential firing and entrainment around any task event, for either dorsolateral or dorsomedial neurons. Among outcome-sensitive FSIs, however, we did find that around goal-reaching, reward discriminative FSIs in the dorsolateral striatum were more likely than nondiscriminative FSIs to be entrained to $5 \mathrm{~Hz}$ theta-band oscillations ( $p=0.0046$, Fisher's Exact test); this relationship failed to reach significance for dorsomedial FSIs ( $p=0.1411$, Fisher's Exact test). Thus, FSIs were similar to MSNs in their propensity to discriminate cue, action, and outcome parameters of the task. However, in opposition to the MSNs, it was the FSIs that encoded information related to the trial outcome, rather than modality or turn direction, that were more likely to be synchronized with ongoing theta oscillations during the relevant task event.
Given the small number of TANs recorded and the lack of any clear region-specific patterns of firing, we again combined dorsolateral and dorsomedial TAN populations to examine sensitivity of this subtype to cue modality, turn response, and trial outcome. TANs exhibited patterns of differential firing to these task parameters consistent with those observed for MSNs and FSIs. Modality-sensitive TAN responses $(n=27$ of 39, 69.2\%) were found predominantly after the onset of the conditional cue, turn-sensitive TAN responses ( $n=23$ of 39, 59.0\%) were found following the initiation of turning, and outcome-sensitive TAN responses ( $n=14$ of $39,35.9 \%$ ) were found only around goalreaching. For this small interneuron population, we did not observe any significant interaction between differential firing for any task parameter examined and simultaneous entrainment to theta-band LFP rhythms.

\section{Discussion}

Our findings have implications of two sorts. First, we demonstrate that interneurons in the sensorimotor and associative striatum have contrasting spike activities that are modulated dynamically as animals perform a reward-based navigational task and that these activities undergo learning-related changes. In particular, FSIs in the dorsolateral and dorsomedial striatum exhibit contrasting dynamics that are broadly consistent with those of the simultaneously recorded MSNs in these regions. These findings suggest that both striatal projection neurons that participate directly in cortico-basal ganglia loops, as well as local circuit neurons that modulate striatal output, express systematic learning-related changes of their activity. Second, we demonstrate that the spiking activities of MSNs and FSIs in these sensorimotor and associative striatal networks are entrained to different theta-band frequencies of LFP oscillations. Neurons in the sensorimotor striatum synchronize preferentially to lowtheta $(\sim 5 \mathrm{~Hz})$ oscillations and become increasingly entrained with learning. By contrast, neurons in the associative striatum synchronize to high-theta $(\sim 10 \mathrm{~Hz})$ oscillations and show a reduction in entrainment late in training. Our findings thus suggest that patterns of spike-LFP entrainment in the dorsolateral and dorsomedial striatum are shaped across training stages in conjunction with the development of distinct patterns of ensemble spiking activity in the two striatal sectors. These patterns of entrainment and local network operation at once support the participation of sensorimotor and associative striatal circuits in distinct anatomical and functional networks and provide further evidence that sensorimotor networks may drive habitual behavior after associative control has waned. We propose that the entrainment of sensorimotor and associative striatal districts to different theta-band frequencies, modulated throughout behavioral learning, could enhance the separation of communication channels for these regions, thereby enabling efficient information flow through simultaneously operating cortico-basal ganglia loops.

\section{Region-specific dynamics of patterned spiking activity and LFP entrainment by striatal subtypes}

Neural firing patterns among interneuron populations in the sensorimotor and associative striatum exhibited divergent patterns of spike activity and spike-LFP entrainment, and these patterns evolved across task-time and across learning with region-specific dynamics. In the dorsolateral striatum, FSIs, like MSNs in this region, developed patterned activation emphasizing the action boundaries of the T-maze task. At the same time, single-unit firing among dorsolateral FSIs and MSNs was entrained preferentially to a low-theta oscillation in the LFP. Both 


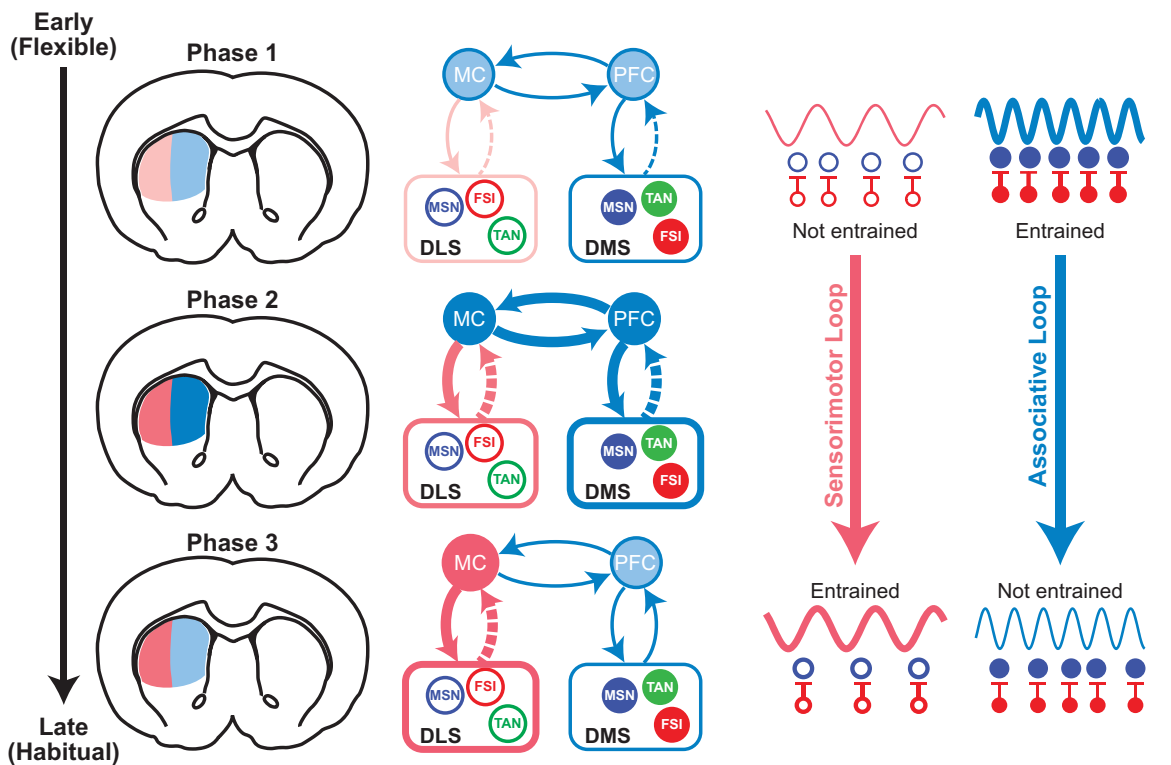

Figure 8. Schematic model illustrating dynamic changes in both ensemble activation and spike-LFP entrainment across learning. Early in training, activity of MSN s conveying information to downstream basal ganglia targets is weakly structured in the sensorimotor striatum, and local spiking activity is weakly entrained to low-theta oscillations. At the same time, MSNs in the associative striatum increase their patterned activation and are strongly entrained to high-theta rhythms, suggesting dominance of the associative trans-striatal loop in the control of motor output. Late in training, the reduction in both structured ensemble activity and high-theta entrainment by the associative striatal network may permit the sensorimotor network to drive the execution of now-habitual behavior via strongly patterned ensemble activity that is entrained to low-theta rhythms. Broken arrows indicate multisynaptic connections from striatum to neocortex through pallidum and thalamus. MC, Motor cortex; PFC, prefrontal cortex; DLS, dorsolateral striatum; DMS, dorsomedial striatum.

of these measures increased across training blocks, reaching their highest expression during the latest phase of training. Thus, both the increase in patterned activation and the increase in spike-LFP entrainment by projection neurons and interneurons in the sensorimotor striatum were associated with the improvement in percentage correct performance and the development of highly stereotyped behavior during learning of the T-maze task.

Results for neuron populations in the dorsomedial striatum were less uniform, but we again found that FSIs exhibited patterned population firing similar to that expressed by local MSNs. Firing rates of the FSIs and MSNs were highest mid-task, but FSIs did not show as much emphasis on the mid-training period (Block B) as the MSN populations did. Dorsomedial FSIs and MSNs were both entrained preferentially to high-theta LFP rhythms; and although the training-related dynamics of this entrainment differed for the two populations, both exhibited low levels of entrainment late in training. This decline in patterned spiking activity and high-theta entrainment among MSNs and FSIs in the dorsomedial striatum occurred during the same latetraining period in which stereotyped maze performance emerged and during which dorsolateral ensemble firing and spike-LFP entrainment was most prominent. Thus, across behavioral training, contrasting patterns of frequency-dependent theta-band entrainment characterized neural activity in the sensorimotor and associative striatum.

Despite these clear regional differences, we found that neither population activity nor entrainment strength in either region varied as a function of the different stimulus modalities in the tasks. As we found previously for MSNs (Thorn et al., 2010), population activity for FSIs and TANs was nearly identical during performance of auditory and tactile versions of the task, and similar proportions of modality-sensitive interneurons were found in dorsolateral and dorsomedial striatum. Moreover, most
FSIs exhibited turn-discriminative activity, but this selectivity held equally for both dorsolateral and dorsomedial regions, again echoing similar results previously reported for MSNs. Nor did we find a regional difference in their responses during rewarded and unrewarded trials. Thus, the ensemble spiking activities that we recorded in the sensorimotor and associative striatal regions were largely indistinguishable with respect to the details of stimulus presentation, motor performance, and reward outcome during acquisition and overtraining on the T-maze. Rather, the distinct patterns of population firing and theta-band entrainment exhibited by the sensorimotor and associative striatal loops appear to reflect characteristics related to the acquisition and performance of the entire task structure as a whole, rather than any trial-specific parameter that we could identify with our techniques.

We did find that entrainment of modality-sensitive MSNs in both striatal regions was enhanced after the presentation of the conditional cue mid-task. Similarly, turn-sensitive MSNs in both regions were more likely to be entrained during the period around turn initiation. Thus, in both regions, striatal neurons that could encode information pertaining to the instruction cue and motor action were also entrained preferentially to region-specific theta-band rhythms during the periods in which the processing of this information was most relevant.

\section{Heterogeneity in dorsomedial FSI populations}

We recorded a large population of FSIs in the dorsomedial striatum that exhibited the short spike durations and high firing rates characteristic of putative parvalbumin-positive (PV-positive) interneurons. Among this group, 15\% exhibited a unique pattern of task-related firing: they underwent long periods of silence midtask, bounded by periods of sustained high firing at the beginning and end of trial-time. The complete absence of activity for up to several seconds has not, to our knowledge, been previously reported for putative or identified PV-positive striatal neurons in vitro or in vivo. Novel putative interneuron subtypes continue to be discovered in the striatum (Ibanez-Sandoval et al., 2011; Tepper et al., 2010). The small number and unusual firing properties of our "task-bracketing" FSIs suggest that they may correspond to one of these newly identified populations. We also note that a higher percentage of neurons in this study were classified as FSIs in the dorsomedial striatum than in the dorsolateral striatum, a result at odds with the increase in the number of PV-positive striatal interneurons along the ventromedial to dorsolateral axis (Graybiel, 1990; Kreitzer, 2009). Although a large fraction of FSIs that we identified are likely to be PV-positive, further cell-type identification is clearly needed.

\section{Theta-band entrainment and differential functional specialization of sensorimotor and associative networks, including hippocampal networks}

Our findings demonstrate that, despite the broad-band coherence between theta-band LFPs recorded in the dorsolateral and 
dorsomedial striatum (DeCoteau et al., 2007a), neurons in these two regions are distinguished by their entrainment to distinct theta-band frequencies. Entrainment of ventral and medial striatal neurons to hippocampal theta rhythm or to locally generated oscillations in the theta frequency range has been documented (Tabuchi et al., 2000; Berke et al., 2004; DeCoteau et al., 2007a). However, reports of theta-band entrainment among dorsolateral neurons are less consistent (Berke et al., 2004; Schmitzer-Torbert and Redish, 2008; Kimchi et al., 2009). Here, we demonstrate that both low-theta and high-theta entrainments can exist within the same animal in simultaneously recorded striatal populations and that the frequency of entrainment is specific to different striatal regions: $\sim 5 \mathrm{~Hz}$ for the sensorimotor dorsolateral striatum and $\sim 10 \mathrm{~Hz}$ for the associative dorsomedial striatum.

These specific entrainment frequencies could reflect the functional specializations thought to characterize these two striatal regions. In the dorsolateral striatum, a subset of "rhythmic bursty" neurons were not only entrained to low-theta $\sim 5 \mathrm{~Hz}$ oscillations, but their burst frequency was also highly correlated with running speed. Evidence from our video tracker data suggested that periodic foot placements during mid-maze running occur at a similar $2.5-5 \mathrm{~Hz}$ rate once asymptotic running performance is reached. These findings suggest that the dorsolateral striatum, strongly interconnected with sensorimotor corticothalamic circuitry, operates at a frequency relevant to the patterned motor behavior of the animals.

In sharp contrast, the dorsomedial striatal neurons became entrained to a higher-frequency, $\sim 10 \mathrm{~Hz}$, theta-band oscillation. The anterior dorsomedial striatum receives input from prelimbic and infralimbic regions of the neocortex (McGeorge and Faull, 1989; Vertes, 2004; Voorn et al., 2004), which in turn are interconnected with the hippocampal formation (Hoover and Vertes, 2007). This network must be intact for accurate performance of spatial working memory and spatial reversal tasks (Ragozzino et al., 1998; Kesner and Gilbert, 2006; Brown et al., 2010) but is distinct from the posterior dorsomedial striatal network shown to support action-outcome encoding (Yin et al., 2005; Corbit and Janak, 2010; Stalnaker et al., 2012). Our group has found neural plasticity throughout the anterior network during learning (Smith and Graybiel, 2013), as well as strong theta-band coherence and cross-frequency coupling between striatal and hippocampal circuitry during T-maze performance (DeCoteau et al., 2007a; Tort et al., 2008). Combined, these findings suggest that the anterior dorsomedial striatum is part of a larger associative network that may include the hippocampal formation and that the theta-band entrainment we have observed might be relevant to the transmission of spatial and/or contextual information throughout the network during sensorimotor learning.

\section{Implications for trans-striatal communication}

A major question raised by our findings is how these different entrainment characteristics relate to the behavioral learning that occurs during the prolonged recording periods of our experiments. Our findings again emphasize the importance of the plasticity of rhythmic entrainment across learning (DeCoteau et al., 2007a). We observed a clear contrast in neural firing patterns and spike-LFP entrainment between simultaneously operating dorsolateral and dorsomedial striatal loops during the acquisition and overtraining on our T-maze task that led to habit-like performance. The learning-related dynamics within these regions demonstrate that the associative network reduced its patterned firing and its high-theta band spike-field entrainment late in training, at the same time that the sensorimotor network strengthened both its low-theta band entrainment and its patterned ensemble activity. The distinct learning-related dynamics and theta-band entrainment patterns that we document for the sensorimotor and associative regions thus support the hypothesis that habitual control of behavior by the sensorimotor striatum can emerge only after competing activation of associative striatal networks has waned (Thorn et al., 2010).

Collectively, these findings reinforce the idea that multiple striatal networks can be simultaneously active, interacting cooperatively or competitively to control behavior, and that this control engages frontal and hippocampal networks. We suggest as a working hypothesis that the network entrainment patterns we describe here could be critical to this learning process (Fig. 8). Theta-band coherence between primary motor cortex and dorsal striatum has been shown to increase during sensorimotor learning, selectively in corticostriatal neurons that control behavioral output (Koralek et al., 2013). Ongoing theta-band rhythms have also been shown to form the base for phase-amplitude coupling across multiple oscillatory rhythms in multiple regions (Tort et al., 2008; Canolty and Knight, 2010; Belluscio et al., 2012). Evidence from our own laboratory and others suggests that these low-frequency LFP oscillations could coordinate communication across brain regions (DeCoteau et al., 2007a; Tort et al., 2008; Canolty and Knight, 2010; Fujisawa and Buzsáki, 2011). Thus, the simultaneous entrainment of sensorimotor and associative striatal networks to different specific theta-band LFP rhythms, as shown here, is likely to affect communication with broader cortical and hippocampal networks as well. Based on these results, we suggest that sensorimotor and associative trans-striatal networks operate on separate communication channels throughout sensorimotor task acquisition and overtraining. In this view, stronger entrainment gained through learning could enhance the efficiency of information transfer throughout a given network, and this increased efficiency could in turn lead to more efficient control over behavioral output. The heightened low-theta entrainment of dorsolateral striatal ensembles, along with the reduced high-theta entrainment of dorsomedial striatal ensembles during the same training period, could thus reflect a core mechanism by which flexible associative-driven control gives way to habitual sensorimotor-driven control as learning occurs.

\section{References}

Alexander GE, DeLong MR, Strick PL (1986) Parallel organization of functionally segregated circuits linking basal ganglia and cortex. Annu Rev Neurosci 9:357-381. CrossRef Medline

Aosaki T, Kimura M, Graybiel AM (1995) Temporal and spatial characteristics of tonically active neurons of the primate's striatum. J Neurophysiol 73:1234-1252. Medline

Balleine BW, O'Doherty JP (2010) Human and rodent homologies in action control: corticostriatal determinants of goal-directed and habitual action. Neuropsychopharmacology 35:48-69. CrossRef Medline

Barnes TD, Kubota Y, Hu D, Jin DZ, Graybiel AM (2005) Activity of striatal neurons reflects dynamic encoding and recoding of procedural memories. Nature 437:1158-1161. CrossRef Medline

Belluscio MA, Mizuseki K, Schmidt R, Kempter R, Buzsáki G (2012) Crossfrequency phase-phase coupling between theta and gamma oscillations in the hippocampus. J Neurosci 32:423-435. CrossRef Medline

Berke JD (2009) Fast oscillations in cortical-striatal networks switch frequency following rewarding events and stimulant drugs. Eur J Neurosci 30:848-859. CrossRef Medline

Berke JD, Okatan M, Skurski J, Eichenbaum HB (2004) Oscillatory entrainment of striatal neurons in freely moving rats. Neuron 43:883-896. CrossRef Medline

Brown HD, Baker PM, Ragozzino ME (2010) The parafascicular thalamic nucleus concomitantly influences behavioral flexibility and dorsomedial 
striatal acetylcholine output in rats. J Neurosci 30:14390-14398. CrossRef Medline

Burkhardt JM, Jin X, Costa RM (2009) Dissociable effects of dopamine on neuronal firing rate and synchrony in the dorsal striatum. Front Integr Neurosci 3:28. CrossRef Medline

Buzsáki G, Anastassiou CA, Koch C (2012) The origin of extracellular fields and currents: EEG, ECoG, LFP and spikes. Nat Rev Neurosci 13:407-420. CrossRef Medline

Canolty RT, Knight RT (2010) The functional role of cross-frequency coupling. Trends Cogn Sci 14:506-515. CrossRef Medline

Corbit LH, Janak PH (2010) Posterior dorsomedial striatum is critical for both selective instrumental and pavlovian reward learning. Eur J Neurosci 31:1312-1321. CrossRef Medline

DeCoteau WE, Thorn C, Gibson DJ, Courtemanche R, Mitra P, Kubota Y, Graybiel AM (2007a) Learning-related coordination of striatal and hippocampal theta rhythms during acquisition of a procedural maze task. Proc Natl Acad Sci U S A 104:5644-5649. CrossRef Medline

DeCoteau WE, Thorn C, Gibson DJ, Courtemanche R, Mitra P, Kubota Y, Graybiel AM (2007b) Oscillations of local field potentials in the rat dorsal striatum during spontaneous and instructed behaviors. J Neurophysiol 97:3800-3805. CrossRef Medline

Emondi AA, Rebrik SP, Kurgansky AV, Miller KD (2004) Tracking neurons recorded from tetrodes across time. J Neurosci Methods 135:95-105. CrossRef Medline

Fujisawa S, Buzsáki G (2011) A 4 Hz oscillation adaptively synchronizes prefrontal, VTA, and hippocampal activities. Neuron 72:153-165. CrossRef Medline

Gatev P, Darbin O, Wichmann T (2006) Oscillations in the basal ganglia under normal conditions and in movement disorders. Mov Disord 21: 1566-1577. CrossRef Medline

Goldberg JA, Reynolds JN (2011) Spontaneous firing and evoked pauses in the tonically active cholinergic interneurons of the striatum. Neuroscience 198:27-43. CrossRef Medline

Graybiel AM (1990) Neurotransmitters and neuromodulators in the basal ganglia. Trends Neurosci 13:244-254. CrossRef Medline

Haber SN (2003) The primate basal ganglia: parallel and integrative networks. J Chem Neuroanat 26:317-330. CrossRef Medline

Hoover WB, Vertes RP (2007) Anatomical analysis of afferent projections to the medial prefrontal cortex in the rat. Brain Struct Funct 212:149-179. CrossRef Medline

Howe MW, Atallah HE, McCool A, Gibson DJ, Graybiel AM (2011) Habit learning is associated with major shifts in frequencies of oscillatory activity and synchronized spike firing in striatum. Proc Natl Acad Sci U S A 108:16801-16806. CrossRef Medline

Ibanez-Sandoval O, Tecuapetla F, Unal B, Shah F, Koós T, Tepper JM (2011) A novel functionally distinct subtype of striatal neuropeptide $\mathrm{Y}$ interneuron. J Neurosci 31:16757-16769. CrossRef Medline

Kalenscher T, Lansink CS, Lankelma JV, Pennartz CM (2010) Rewardassociated gamma oscillations in ventral striatum are regionally differentiated and modulate local firing activity. J Neurophysiol 103:1658-1672. CrossRef Medline

Kemp JM, Powell TP (1971) The structure of the caudate nucleus of the cat: light and electron microscopy. Philos Trans R Soc Lond B Biol Sci 262: 383-401. CrossRef Medline

Kesner RP, Gilbert PE (2006) The role of the medial caudate nucleus, but not the hippocampus, in a matching-to sample task for a motor response. Eur J Neurosci 23:1888-1894. CrossRef Medline

Kimchi EY, Torregrossa MM, Taylor JR, Laubach M (2009) Neuronal correlates of instrumental learning in the dorsal striatum. J Neurophysiol 102:475-489. CrossRef Medline

Kojima S, Kao MH, Doupe AJ (2013) Task-related "cortical" bursting depends critically on basal ganglia input and is linked to vocal plasticity. Proc Natl Acad Sci U S A 110:4756-4761. CrossRef Medline

Koralek AC, Costa RM, Carmena JM (2013) Temporally precise cellspecific coherence develops in corticostriatal networks during learning. Neuron 79:865-872. CrossRef Medline

Kreitzer AC (2009) Physiology and pharmacology of striatal neurons. Annu Rev Neurosci 32:127-147. CrossRef Medline

Logothetis NK (2002) The neural basis of the blood-oxygen-level- dependent functional magnetic resonance imaging signal. Philos Trans $\mathrm{R}$ Soc Lond B Biol Sci 357:1003-1037. CrossRef Medline

Malhotra S, Cross RW, van der Meer MA (2012) Theta phase precession beyond the hippocampus. Rev Neurosci 23:39-65. CrossRef Medline

McGeorge AJ, Faull RL (1989) The organization of the projection from the cerebral cortex to the striatum in the rat. Neuroscience 29:503-537. CrossRef Medline

Morris G, Arkadir D, Nevet A, Vaadia E, Bergman H (2004) Coincident but distinct messages of midbrain dopamine and striatal tonically active neurons. Neuron 43:133-143. CrossRef Medline

Packard MG (2009) Exhumed from thought: basal ganglia and response learning in the plus-maze. Behav Brain Res 199:24-31. CrossRef Medline

Pesaran B, Pezaris JS, Sahani M, Mitra PP, Andersen RA (2002) Temporal structure in neuronal activity during working memory in macaque parietal cortex. Nat Neurosci 5:805-811. CrossRef Medline

Ragozzino ME, Adams S, Kesner RP (1998) Differential involvement of the dorsal anterior cingulate and prelimbic-infralimbic areas of the rodent prefrontal cortex in spatial working memory. Behav Neurosci 112:293303. CrossRef Medline

Schmitzer-Torbert NC, Redish AD (2008) Task-dependent encoding of space and events by striatal neurons is dependent on neural subtype. Neuroscience 153:349-360. CrossRef Medline

Schmitzer-Torbert N, Jackson J, Henze D, Harris K, Redish AD (2005) Quantitative measures of cluster quality for use in extracellular recordings. Neuroscience 131:1-11. CrossRef Medline

Sharott A, Moll CK, Engler G, Denker M, Grün S, Engel AK (2009) Different subtypes of striatal neurons are selectively modulated by cortical oscillations. J Neurosci 29:4571-4585. CrossRef Medline

Smith KS, Graybiel AM (2013) A dual operator view of habitual behavior reflecting cortical and striatal dynamics. Neuron 79:361-374. CrossRef Medline

Stalnaker TA, Calhoon GG, Ogawa M, Roesch MR, Schoenbaum G (2012) Reward prediction error signaling in posterior dorsomedial striatum is action specific. J Neurosci 32:10296-10305. CrossRef Medline

Tabuchi ET, Mulder AB, Wiener SI (2000) Position and behavioral modulation of synchronization of hippocampal and accumbens neuronal discharges in freely moving rats. Hippocampus 10:717-728. CrossRef Medline

Tepper JM, Tecuapetla F, Koós T, Ibáñez-Sandoval O (2010) Heterogeneity and diversity of striatal gabaergic interneurons. Front Neuroanat 4:150. CrossRef Medline

Thorn CA, Atallah H, Howe M, Graybiel AM (2010) Differential dynamics of activity changes in dorsolateral and dorsomedial striatal loops during learning. Neuron 66:781-795. CrossRef Medline

Tort AB, Kramer MA, Thorn C, Gibson DJ, Kubota Y, Graybiel AM, Kopell NJ (2008) Dynamic cross-frequency couplings of local field potential oscillations in rat striatum and hippocampus during performance of a T-maze task. Proc Natl Acad Sci U S A 105:20517-20522. CrossRef Medline

van der Meer MA, Redish AD (2009) Low and high gamma oscillations in rat ventral striatum have distinct relationships to behavior, reward, and spiking activity on a learned spatial decision task. Front Integr Neurosci 3:9. CrossRef Medline

Vertes RP (2004) Differential projections of the infralimbic and prelimbic cortex in the rat. Synapse 51:32-58. CrossRef Medline

Vinck M, Battaglia FP, Womelsdorf T, Pennartz C (2012) Improved measures of phase-coupling between spikes and the local field potential. J Comp Neurosci 33:53-75. CrossRef Medline

Voorn P, Vanderschuren LJ, Groenewegen HJ, Robbins TW, Pennartz CM (2004) Putting a spin on the dorsal-ventral divide of the striatum. Trends Neurosci 27:468-474. CrossRef Medline

Yin HH, Knowlton BJ, Balleine BW (2006) Inactivation of dorsolateral striatum enhances sensitivity to changes in the action-outcome contingency in instrumental conditioning. Behav Brain Res 166:189-196. CrossRef Medline

Yin HH, Ostlund SB, Knowlton BJ, Balleine BW (2005) The role of the dorsomedial striatum in instrumental conditioning. Eur J Neurosci 22: 513-523. CrossRef Medline

Yin HH, Mulcare SP, Hilário MR, Clouse E, Holloway T, Davis MI, Hansson AC, Lovinger DM, Costa RM (2009) Dynamic reorganization of striatal circuits during the acquisition and consolidation of a skill. Nat Neurosci 12:333-341. CrossRef Medline 